JOURNAL OF THE

AMERICAN MATHEMATICAL SOCIETY

Volume 13, Number 2, Pages 343-370

S 0894-0347(99)00324-0

Article electronically published on November 23, 1999

\title{
RIGIDITY OF CRITICAL CIRCLE MAPPINGS II
}

\author{
EDSON DE FARIA AND WELINGTON DE MELO
}

\section{INTRODUCTION}

A celebrated rigidity theorem of M. Herman [5] states that any two smooth diffeomorphisms of the circle with the same Diophantine rotation number are smoothly conjugate. Herman used purely real-variable techniques to prove this theorem. In recent years, other fundamental rigidity results have been established in the context of one-dimensional dynamics with the help of complex-analytic techniques. Thus, in the context of circle diffeomorphisms, J.-C. Yoccoz [16] used conformal surgery to describe the optimal class of rotation numbers for which Herman's theorem holds in the real-analytic category. In the context of interval maps, D. Sullivan [13] and C. McMullen [10] used a combination of real-variable techniques with deep results from Teichmüller theory and hyperbolic geometry to establish the rigidity of the Cantor attractor of a unimodal map with bounded combinatorics. The conjugacy between two such maps with the same combinatorics is shown to be $C^{1+\alpha}$ for some $\alpha>0$ depending only on the combinatorics. In sharp contrast with the case of circle diffeomorphisms, in this case one does not expect the conjugacy to be much better than $C^{1}$, even when the unimodal maps are analytic.

In this paper we prove the following strong rigidity theorem for real-analytic critical circle maps.

Theorem 1.1. Any two real-analytic critical circle maps with the same irrational rotation number $\rho$ of bounded type are $C^{1+\alpha}$ conjugate for some $0<\alpha<1$ depending only on the least upper-bound of the coefficients of the continued fraction expansion of $\rho$.

The proof of this theorem uses both real and complex-analytic tools. We use the real a-priori bounds of Świątek and Herman (see [14, 6] and [3]) in the form stated in $\S 2$. The complex-analytic part we develop here combines some of the powerful new ideas on conformal rigidity and renormalization recently developed by McMullen in [9, [10], 11] with the basic theory of holomorphic commuting pairs introduced in [2] and a generalization of the Lyubich-Yampolsky approach to the complex bounds given in [8, [15].

The general outline of the paper is as follows. First we prove that after a finite number of renormalizations, every real-analytic critical circle map with arbitrary irrational rotation number gives rise to a holomorphic commuting pair with good

Received by the editors November 9, 1998 and, in revised form, September 20, 1999.

2000 Mathematics Subject Classification. Primary 37F25; Secondary 37E10, 30D05, 37F40.

Key words and phrases. Holomorphic pairs, complex bounds, uniform twist, rigidity.

This work has been partially supported by the Pronex Project on Dynamical Systems, by FAPESP Grant 95/3187-4 and by CNPq Grant 30.1244/86-3. 
geometric bounds. Next, we prove that such a holomorphic pair (with good geometric control) possesses McMullen's uniform twisting property [10]. We also prove that its critical point is $\delta$-deep for some universal $\delta>0$. The general machinery developed by McMullen in [9], [10] permits us to deduce from these facts that the conjugacy between any two real-analytic critical circle maps with rotation number of type bounded by $N$ is $C^{1+\varepsilon(N)}$-conformal at the critical point. This implies that the successive renormalizations of both circle maps around their critical points are converging together exponentially fast in the $C^{0}$ sense (even $C^{1+\beta}$ for some $\beta>0$ ), with rate of convergence given by a universal function of $\varepsilon$. By a general proposition proved in [3], this implies at last that the conjugacy between both maps is $C^{1+\alpha(N)}$ for some $\alpha(N)>0$. Although in our proof the Hölder exponent depends on $N$, we believe that this should not be the case.

It is to be noted that in [3] we constructed $C^{\infty}$ counterexamples (even with Diophantine rotation numbers) to this form of $C^{1+\alpha}$ rigidity. One may ask whether such counterexamples exist in the analytic category. We do not know the answer to this question. Nevertheless, we conjecture that in the general case the conjugacy is always $C^{1+\alpha}$ at the critical point, for some universal $\alpha$ independent of the rotation number. A possible approach to this conjecture is to use M. Lyubich's recent work on quadratic-like maps and universality of unimodal maps, but it is still unclear at this time how to adapt his methods to holomorphic pairs and critical circle maps.

\section{Preliminaries}

In this section, we introduce some basic concepts and notations. We denote by $|I|$ the length of an interval $I$ on the line or the circle. We use $\operatorname{dist}(\cdot, \cdot)$ for Euclidean distance, and $\operatorname{diam}(\cdot)$ for Euclidean diameter. All bounds achieved in this paper will depend on certain a-priori constants (which can ultimately be traced back to the real a-priori bounds given by Theorem 2.1 below). In this context, two positive quantities $a$ and $b$ are said to be comparable if there exists a constant $C>1$ depending only on the a-priori bounds such that $C^{-1} b \leq a \leq C b$. We write $a \asymp b$ to denote that $a$ is comparable to $b$.

Critical circle maps. We identify the unit circle $S^{1}$ with the one-dimensional torus $\mathbb{R} / \mathbb{Z}$. A homeomorphism $f: S^{1} \rightarrow S^{1}$ which is at least $C^{1}$ and satisfies $f^{\prime}(c)=0$ and $f^{\prime}(x) \neq 0$ for all $x \neq c$ is called a critical circle map. The point $c$ is the critical point of $f$. In this paper $f$ will be real-analytic, and the critical point will be cubic: this means that near $c$ we have $f=\phi \circ Q \circ \psi$, where $\phi, \psi$ are real analytic diffeomorphisms and $Q$ is the map $x \mapsto x^{3}$.

We are interested in the geometry of orbits of $f$ only when there are no periodic points. In this case the rotation number of $f$ is irrational and can be represented as an infinite continued fraction

$$
\rho(f)=\left[a_{0}, a_{1}, \ldots, a_{n}, \ldots\right]=\frac{1}{a_{0}+\frac{1}{a_{1}+\frac{1}{\frac{\cdots}{a_{n}+\frac{1}{\cdots}}}} .}
$$

When the partial quotients $a_{n}$ are bounded, we say that $\rho(f)$ is a number of bounded type. We also refer to $\sup a_{n}$ as the combinatorial type of $\rho(f)$. 
The denominators of the convergents of $\rho(f)$, defined recursively by $q_{0}=1$, $q_{1}=a_{0}$ and $q_{n+1}=a_{n} q_{n}+q_{n-1}$ for all $n \geq 1$, are the closest return times of the orbit of any point to itself. We denote by $\Delta_{n}$ the closed interval containing $c$ whose endpoints are $f^{q_{n}}(c)$ and $f^{q_{n+1}}(c)$. We also let $I_{n} \subseteq \Delta_{n}$ be the closed interval whose endpoints are $c$ and $f^{q_{n}}(c)$. Observe that $\Delta_{n}=I_{n} \cup I_{n+1}$. The most important fact to remember when studying the geometry of a circle map is that for each $n$ the collection of intervals

$$
\mathcal{P}_{n}=\left\{I_{n}, f\left(I_{n}\right), \ldots, f^{q_{n+1}-1}\left(I_{n}\right)\right\} \cup\left\{I_{n+1}, f\left(I_{n+1}\right), \ldots, f^{q_{n}-1}\left(I_{n+1}\right)\right\}
$$

constitutes a partition of the circle (modulo endpoints), called dynamical partition of level $n$ of the map $f$. Note that, for all $n, \mathcal{P}_{n+1}$ is a refinement of $\mathcal{P}_{n}$.

Of course, these definitions make sense for an arbitrary homeomorphism of the circle. For a rigid rotation, we have $\left|I_{n}\right|=a_{n+1}\left|I_{n+1}\right|+\left|I_{n+2}\right|$. Therefore, if $a_{n+1}$ is very large, then $I_{n}$ is much longer than $I_{n+1}$. It is a remarkable fact, first proved by Świątek and Herman, that this never happens for a critical circle map! The dynamical partitions $\mathcal{P}_{n}$ have bounded geometry, in the sense that adjacent atoms have comparable lengths.

Theorem 2.1 (The real bounds). Let $f: S^{1} \rightarrow S^{1}$ be a critical circle map with arbitrary irrational rotation number. There exists $n_{0}=n_{0}(f)$ such that for all $n \geq n_{0}$ and every pair $I, J$ of adjacent atoms of $\mathcal{P}_{n}$ we have $K^{-1}|J| \leq|I| \leq K|J|$, where $K>1$ is a universal constant.

These real a-priori bounds are essential in all the estimates that we perform in this paper. For proofs, see [4] and $\S 3$ of [3]. An important consequence of this theorem is the fact that every critical circle map with rotation number of bounded type is conjugate to the rotation with the same rotation number by a quasisymmetric homeomorphism. Another consequence is the following lemma.

Lemma 2.2. Let $f$ be a critical circle map with arbitrary rotation number, let $n \geq$ 1 , and let $J_{-i}=f^{q_{n+1}-i}\left(I_{n}\right)$ for $0 \leq i<q_{n+1}$. Given $m<n$, let $i_{1}<i_{2}<\cdots<i_{\ell}$ be the moments in the backward orbit $\left\{J_{-i}\right\}$ of $I_{n}$ before the first return to $I_{m+1}$ such that $J_{-i_{k}} \subseteq I_{m}$. Then $\ell=a_{m+1}$, and we have

$$
J_{-i_{k}} \subseteq f^{q_{m}+\left(a_{m+1}-k+1\right) q_{m+1}}\left(I_{m+1}\right) .
$$

Moreover, given an integer $M \geq 1$, there exists $C_{M}>1$ (depending only on $M$ ) such that for all sufficiently large $n$ we have $C_{M}^{-1}\left|I_{n}\right| \leq\left|J_{-i_{k}}\right| \leq C_{M}\left|I_{n}\right|$, provided $1 \leq k \leq M$ or $a_{m+1}-M+1 \leq k \leq a_{m+1}$.

Proof. The largest $j<q_{n+1}$ such that $f^{j}\left(I_{n}\right) \subseteq I_{m+1}$ is easily computed as $j=$ $q_{n+1}-q_{m+2}$. Since $q_{m+2}=q_{m}+a_{m+1} q_{m+1}$, there are exactly $a_{m+1}$ subsequent moments $j<i<q_{n+1}$ such that $f^{i}\left(I_{n}\right) \subseteq I_{m}$. The rest follows from Theorem 2.1 and the Koebe distortion principle (see [12]).

Commuting pairs and renormalization. Let $f$ be a critical circle map as before, and let $n \geq 1$. The first return map $f_{n}: \Delta_{n} \rightarrow \Delta_{n}$ to $\Delta_{n}=I_{n} \cup I_{n+1}$, called the $n$-th renormalization of $f$ without rescaling, is determined by a pair of maps, namely $f^{q_{n}}: I_{n+1} \rightarrow \Delta_{n}$ and $f^{q_{n+1}}: I_{n} \rightarrow \Delta_{n}$. This pair of maps is what we call a critical commuting pair. Note that $f_{n}$ has a well-defined rotation number (identifying the endpoints of $\Delta_{n}$, we recover a circle map). Each $f_{n+1}$ is by definition the renormalization without rescaling of $f_{n}$. These objects can be 
normalized to unit size to yield the $n$-th renormalization of $f$, denoted $\mathcal{R}^{n}(f)$, in the following way. Consider the exponential covering map exp : $\mathbb{R} \rightarrow S^{1}$ given by $\exp (x)=e^{2 \pi i x}$, and let $T$ denote the translation $x \mapsto x+1$. We assume that the critical point of $f$ is $c=1 \in S^{1}$. There is a unique lift $\hat{f}: \mathbb{R} \rightarrow \mathbb{R}$ of $f$ to the real line through the exponential map such that $0 \leq \hat{f}(0)<1$; henceforth referred to as the standard lift of $f$. Let $\widetilde{I}_{n} \subset \mathbb{R}$ be the interval whose endpoints are 0 and $\lambda_{n}=T^{-p_{n}} \circ \hat{f}^{q_{n}}(0)$, where $p_{n}$ is the numerator of the $n$-th convergent of $\rho(f)$. Note that $\exp \left(\widetilde{I}_{n}\right)=I_{n}$. Write $\widetilde{\Delta}_{n}=\widetilde{I}_{n} \cup \widetilde{I}_{n+1}$. Let $\xi_{n}=T^{-p_{n}} \circ \hat{f}^{q_{n}}: \widetilde{I}_{n+1} \rightarrow \widetilde{\Delta}_{n}$ and $\eta_{n}=T^{-p_{n+1}} \circ \hat{f}^{q_{n+1}}: \widetilde{I}_{n} \rightarrow \widetilde{\Delta}_{n}$. This pair of maps projects down to the pair $\left(f^{q_{n}}, f^{q_{n+1}}\right)$ on the circle. Finally, let $\hat{\xi}_{n}=\Lambda_{n}^{-1} \circ \xi_{n} \circ \Lambda_{n}$ and $\hat{\eta}_{n}=\Lambda_{n}^{-1} \circ \eta_{n} \circ \Lambda_{n}$, where $\Lambda_{n}$ is the linear map $x \mapsto \lambda_{n} x$. The resulting critical commuting pair $\left(\hat{\xi}_{n}, \hat{\eta}_{n}\right)$ is by definition the (normalized) $n$-th renormalization $\mathcal{R}^{n}(f)$. For more on commuting pairs, including the abstract definition, see [3].

Holomorphic pairs. The concept of holomorphic commuting pair was introduced in [2, and will play a crucial role in this paper. We recall the definition and some of the relevant facts about these objects; henceforth called simply holomorphic pairs. Assume we are given a configuration of four simply-connected domains $\mathcal{O}_{\xi}, \mathcal{O}_{\eta}, \mathcal{O}_{\nu}, \mathcal{V}$ in the complex plane, called a bowtie, such that

(a) each $\mathcal{O}_{\gamma}$ is a Jordan domain whose closure is contained in $\mathcal{V}$;

(b) we have $\overline{\mathcal{O}}_{\xi} \cap \overline{\mathcal{O}}_{\eta}=\{0\} \subseteq \mathcal{O}_{\nu}$;

(c) the sets $\mathcal{O}_{\xi} \backslash \mathcal{O}_{\nu}, \mathcal{O}_{\eta} \backslash \mathcal{O}_{\nu}, \mathcal{O}_{\nu} \backslash \mathcal{O}_{\xi}$ and $\mathcal{O}_{\nu} \backslash \mathcal{O}_{\eta}$ are non-empty and connected.

Definition. A holomorphic pair $\Gamma$ with domain $\mathcal{U}=\mathcal{O}_{\xi} \cup \mathcal{O}_{\eta} \cup \mathcal{O}_{\nu}$ and co-domain $\mathcal{V}$ is the dynamical system generated by three holomorphic maps $\xi: \mathcal{O}_{\xi} \rightarrow \mathbb{C}$, $\eta: \mathcal{O}_{\eta} \rightarrow \mathbb{C}$ and $\nu: \mathcal{O}_{\nu} \rightarrow \mathbb{C}$ satisfying the following conditions (see Figure 1).

$\left(\mathrm{H}_{1}\right)$ Both $\xi$ and $\eta$ are univalent onto $\mathcal{V} \cap \mathbb{C}\left(\xi\left(J_{\xi}\right)\right)$ and $\mathcal{V} \cap \mathbb{C}\left(\eta\left(J_{\eta}\right)\right)$ respectively, where $J_{\xi}=\mathcal{O}_{\xi} \cap \mathbb{R}$ and $J_{\eta}=\mathcal{O}_{\eta} \cap \mathbb{R}$. (Notation: $\mathbb{C}(I)=(\mathbb{C} \backslash \mathbb{R}) \cup I$.)

$\left(\mathrm{H}_{2}\right)$ The map $\nu$ is a 3 -fold branched cover onto $\mathcal{V} \cap \mathbb{C}\left(\nu\left(J_{\nu}\right)\right)$, where $J_{\nu}=\mathcal{O}_{\nu} \cap \mathbb{R}$, with a unique critical point at 0 .

$\left(\mathrm{H}_{3}\right)$ We have $\mathcal{O}_{\xi} \ni \eta(0)<0<\xi(0) \in \mathcal{O}_{\eta}$, and the restrictions $\xi \mid[\eta(0), 0]$ and $\eta \mid[0, \xi(0)]$ constitute a critical commuting pair.

$\left(\mathrm{H}_{4}\right)$ Both $\xi$ and $\eta$ extend holomorphically to a neighborhood of zero, and we have $\xi \circ \eta(z)=\eta \circ \xi(z)=\nu(z)$ for all $z$ in that neighborhood.

$\left(\mathrm{H}_{5}\right)$ There exists an integer $m \geq 1$, called the height of $\Gamma$, such that $\xi^{m}(a)=\eta(0)$, where $a$ is the left endpoint of $J_{\xi}$; moreover, $\eta(b)=\xi(0)$, where $b$ is the right endpoint of $J_{\eta}$.

The interval $J=[a, b]$ is called the long dynamical interval of $\Gamma$, whereas $\Delta=$ $[\eta(0), \xi(0)]$ is the short dynamical interval of $\Gamma$. They are both forward invariant under the dynamics. The rotation number of $\Gamma$ is by definition the rotation number of the critical commuting pair of $\Gamma$ (condition $\left.\mathrm{H}_{3}\right)$.

Examples of holomorphic pairs with arbitrary rotation number and arbitrary height were carefully constructed in $\S 4$ of [2], with the help of the family of entire maps given by

$$
z \mapsto z+\theta-\frac{1}{2 \pi} \sin 2 \pi z
$$

where $\theta$ is real, the so-called Arnold family. Many interesting properties of holomorphic pairs can be proved with these examples at hand. 


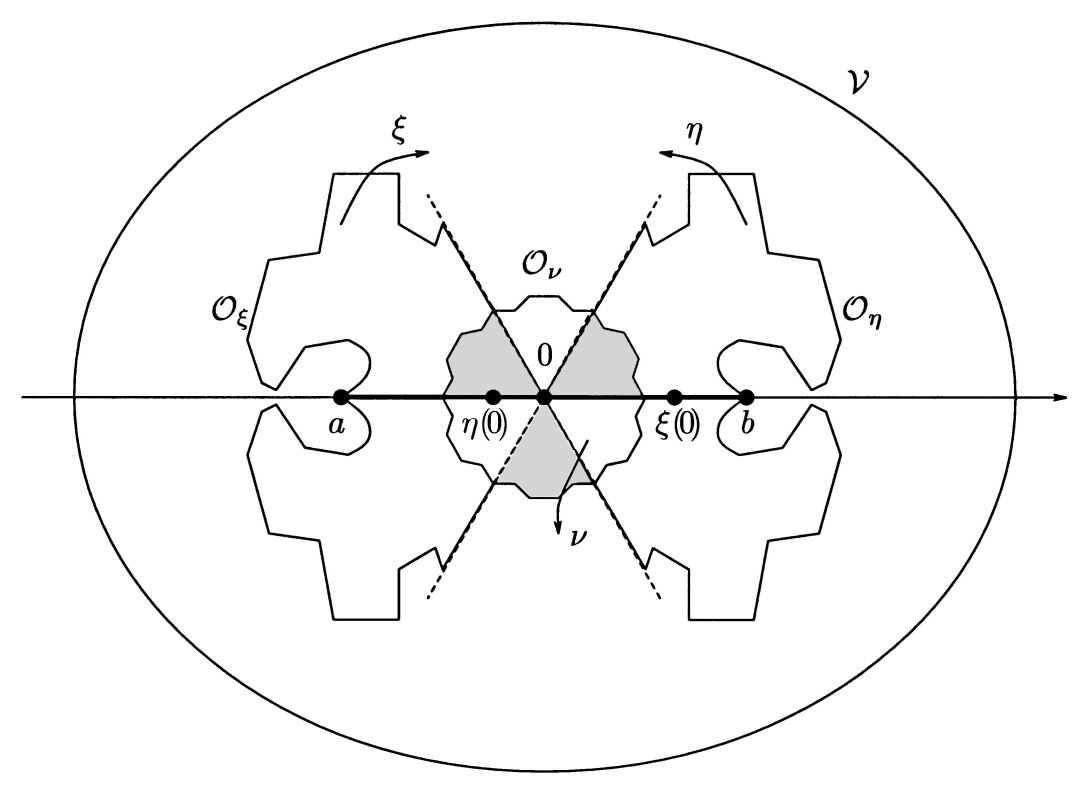

FiguRE 1.

The shadow of a holomorphic pair is the map $F: \mathcal{O}_{\xi} \cup \mathcal{O}_{\eta} \cup \mathcal{O}_{\nu} \rightarrow \mathcal{V}$ given by

$$
F(z)= \begin{cases}\xi(z), & \text { when } z \in \mathcal{O}_{\xi}, \\ \eta(z), & \text { when } z \in \mathcal{O}_{\eta}, \\ \nu(z), & \text { when } z \in \mathcal{O}_{\nu} \backslash\left(\mathcal{O}_{\xi} \cup \mathcal{O}_{\eta}\right) .\end{cases}
$$

The shadow captures the essential dynamical features of a holomorphic pair. It is a simple fact (see [2], Prop. 2.4) that every $\Gamma$-orbit is an $F$-orbit and conversely.

The limit set of a holomorphic pair $\Gamma$, denoted $\mathcal{K}_{\Gamma}$, is the closure of the set of points in $\mathcal{U}$ which never escape from $\mathcal{U}$ under iteration by $F$. The annulus $\mathcal{V} \backslash \mathcal{U}$ is a fundamental domain for the dynamics off the limit set, and for this reason it will be called the fundamental annulus of $\Gamma$.

Holomorphic pairs can be renormalized. In other words, the first renormalization of the critical commuting pair of $\Gamma$ extends in a natural way to a holomorphic pair $\mathcal{R}(\Gamma)$ with the same co-domain $\mathcal{V}$. For the careful construction of $\mathcal{R}(\Gamma)$, see Prop. 2.3 in [2]. There is also a pull-back theorem for holomorphic pairs.

Definition. We say that $\Gamma$ has geometric boundaries if $\partial \mathcal{U}$ and $\partial \mathcal{V}$ are quasicircles.

Theorem 2.3. Let $\Gamma$ and $\Gamma^{\prime}$ be holomorphic pairs with geometric boundaries, and let $h: J \rightarrow J^{\prime}$ be a quasisymmetric conjugacy between $F \mid J$ and $F^{\prime} \mid J^{\prime}$. Then there exists a quasiconformal conjugacy $H: \mathcal{V} \rightarrow \mathcal{V}^{\prime}$ between $\Gamma$ and $\Gamma^{\prime}$ which is an extension of $h$.

For a proof of this theorem, see Th. 3.1 in [2].

Conformal distortion. We will need some classical facts about conformal maps; see [1] and [9]. Consider a univalent map $\phi: \Omega \rightarrow \mathbb{C}$ on a domain $\Omega \subseteq \mathbb{C}$. The 
distortion of $\phi$ on a compact set $E \subseteq \Omega$ is the largest ratio $\left|\phi^{\prime}(z)\right| /\left|\phi^{\prime}(w)\right|$ with $z, w \in E$. When $E$ is convex (a disk in most cases), the distortion on $E$ is bounded by $\exp N_{\phi}(E)$, where $N_{\phi}(E)=\sup _{z \in E}\left|\phi^{\prime \prime}(z)\right| /\left|\phi^{\prime}(z)\right|$ is the total non-linearity of $\phi$ on $E$. The Koebe distortion theorem states that $N_{\phi}(E) \leq 2 / \operatorname{dist}(E, \partial \Omega)$. Koebe's one-quarter theorem states that if $D \subseteq \Omega$ is a disk centered at $z$, then $\phi(D)$ contains a disk centered at $\phi(z)$ of radius $\frac{1}{4} \operatorname{diam}(D)\left|\phi^{\prime}(z)\right|$.

Almost parabolic maps. In order to adapt Yampolsky's argument for the complex bounds in $\S 3$, we will need to know some general facts about complex analytic maps that are very close to maps with a parabolic fixed-point. Given $J \subseteq \mathbb{R}$ and $0<\theta<\pi$, we denote by $\mathbb{P}_{\theta}(J) \subseteq \mathbb{C}$ the set of all $z$ in the complex plane that view $J$ under an angle $\geq \theta$. This set is the Poincaré neighborhood of $J$ in $\mathbb{C}(J)$ with angle $\theta$.

Definition. Let $J \subseteq \mathbb{R}$ be an interval, and let $\theta>0$. A holomorphic univalent map $\phi: \mathbb{P}_{\theta}(J) \rightarrow \mathbb{C}$ is called almost parabolic if the following conditions are satisfied.

(a) $\phi$ is symmetric about the real axis, that is, $\phi(\bar{z})=\overline{\phi(z)}$ for all $z \in \mathbb{P}_{\theta}(J)$.

(b) $\phi \mid J$ is monotone without fixed points.

(c) $\phi$ has positive Schwarzian derivative on $J$.

(d) $J \cap \phi(J)$ is non-empty.

If $\Delta_{\phi}$ is the interval $J \backslash \phi(J)$, the largest $a=a(\phi)>0$ such that $\phi^{a-1}\left(\Delta_{\phi}\right) \subseteq J$ is called the length of $\phi$. The number $\theta=\theta(\phi)$ is the angle of $\phi$.

The most important example of an almost parabolic map is the inverse branch of a high first return $f^{q_{n}}$ of a critical circle map $f$ (or one of its renormalizations), in the situation where $a_{n}$ is large, that is, the rotation number is "almost rational".

Given $0<\sigma<1$, we denote by $\mathcal{F}_{\sigma}$ the family of all almost parabolic maps $\phi$ such that $\left|\Delta_{\phi}\right| \geq \sigma|J|$ and $\left|\phi^{a-1}\left(\Delta_{\phi}\right)\right| \geq \sigma|J|$, and also normalized so that

$$
[0,1]=\Delta_{\phi} \cup \phi\left(\Delta_{\phi}\right) \cup \cdots \cup \phi^{a-1}\left(\Delta_{\phi}\right) .
$$

Now we have the following fundamental lemma due to Yoccoz (see [3] for a proof).

Yoccoz's Lemma. There exists $C_{\sigma}>1$ such that for each $\phi \in \mathcal{F}_{\sigma}$ and for each $0 \leq j \leq a-1$ we have

$$
\frac{1}{C_{\sigma} m(j)^{2}} \leq\left|\phi^{j}\left(\Delta_{\phi}\right)\right| \leq \frac{C_{\sigma}}{m(j)^{2}},
$$

where $m(j)=\min \{j+1, a-j\}$.

Every member of $\mathcal{F}_{\sigma}$ whose length is sufficiently large has two fixed points, symmetric about the real axis. These fixed points are necessarily attracting, due to the positive Schwarzian property of the maps in $\mathcal{F}_{\sigma}$. More precisely, we have the following fact. Let us denote by $\mathbb{H}$ the upper half-plane.

Lemma 2.4. Given $0<\sigma<1$, there exist $C>1, a_{0}>0$ and $\theta_{0}>0$ such that, if $\phi \in \mathcal{F}_{\sigma}$ has length $a=a(\phi)>a_{0}$ and angle $\theta(\phi)<\theta_{0}$, then there exist two attracting fixed points $z_{+} \in \mathbb{H} \cap \operatorname{Dom}(\phi)$ and $z_{-}=\bar{z}_{+}$with

$$
\frac{1}{C a} \leq \operatorname{Im} z_{+} \leq \frac{C}{a}
$$

Moreover, if $\left|z-z_{+}\right| \leq C / a$, then $|z-\phi(z)| \leq C / a^{2}$. 
Proof. The lemma follows from Yoccoz's Lemma, the saddle-node bifurcation and a normality argument.

The family $\mathcal{F}_{\sigma}$ is normal in the sense of Montel, and every limit map not in $\mathcal{F}_{\sigma}$ is a map with a parabolic (indifferent) fixed point on the real axis, whose multiplier is necessarily equal to one.

Lemma 2.5. Given a compact set $W \subset \mathbb{H}$ and an open set $D \supset[0,1]$ in the plane, there exist $N_{*}>0, \theta_{*}>0$ and $a_{*}>0$ with the following property. For each $\phi \in \mathcal{F}_{\sigma}$ such that $a(\phi) \geq a_{*}$ and $\theta(\phi)<\theta_{*}$, the domain of $\phi$ contains $W$, and for each $z \in W$ there exists $n<N_{*}$ such that $\phi^{n}(z) \in D$.

Proof. If the statement is false, we find sequences $N_{k} \rightarrow \infty, a_{k} \rightarrow \infty$ and $\theta_{k} \rightarrow 0$, maps $\phi_{k} \in \mathcal{F}_{\sigma}$ with $\theta\left(\phi_{k}\right)=\theta_{k}$ and $a\left(\phi_{k}\right)=a_{k}$ (whose domains contain $W$ ), and points $z_{k} \in W$ such that $\phi_{k}^{n}\left(z_{k}\right)$, whenever defined, does not belong to $D$ for all $n \leq N_{k}$. Since $\mathcal{F}_{\sigma}$ is normal and $W$ is compact, we may assume that the sequence $\phi_{k}$ converges uniformly on compact subsets of $\mathbb{H}$ to a map $\phi: \mathbb{H} \rightarrow \mathbb{H}$ and that $z_{k} \rightarrow z \in W$. Applying Lemma 2.4 to each $\phi_{k}$, we deduce that $\phi$ has a fixed-point $x_{0} \in[0,1]$. By the Denjoy-Wolff theorem, $\phi^{n}(z) \rightarrow x_{0}$ as $n \rightarrow \infty$. Hence there exists $N$ such that $\phi^{N}(z) \in D$. But then $\phi_{k}^{N}\left(z_{k}\right) \in D$ also, for all sufficiently large $k$, a contradiction.

\section{Complex Bounds For ReAL-ANALYTiC CIRCLE MAPS}

We will extend Yampolsky's proof of complex bounds for circle maps in the Epstein class [15] to the more general case of real-analytic circle maps. More precisely, we will prove the following result.

Theorem 3.1 (The complex bounds). Let $f: S^{1} \rightarrow S^{1}$ be a real-analytic critical circle map with arbitrary irrational rotation number. Then there exists $n_{0}=n_{0}(f)$ such that for all $n \geq n_{0}$ the $n$-th renormalization of $f$ extends to a holomorphic pair with geometric boundaries (see definition in §2) whose fundamental annulus has conformal modulus bounded from below by a universal constant.

The proof of Theorem 3.1 will be given at the end of this section. The main step in the proof is to show that for all sufficiently large $n$ the appropriate inverse branch of $f^{q_{n+1}}$ maps a sufficiently large disk around the $n$-th renormalization domain $I_{n} \cup I_{n+1}$ well within itself.

We consider the unit circle $S^{1}=\mathbb{R} / \mathbb{Z}$ embedded in the infinite cylinder $\mathbb{C} / \mathbb{Z}$, and in all the geometric considerations that follow we use on $\mathbb{C} / \mathbb{Z}$ the conformal metric induced from the standard Euclidean metric $|d z|$ on $\mathbb{C}$ via the exponential map $\exp : \mathbb{C} \rightarrow \mathbb{C} / \mathbb{Z}$ given by $\exp (z)=e^{2 \pi i z}$. Note that $\operatorname{Im} z$ is well defined for every $z \in \mathbb{C} / \mathbb{Z}$ (as the imaginary part of any one of its pre-images under the exponential).

In what follows, we will fix $f: S^{1} \rightarrow S^{1}$ as in the statement of Theorem 3.1. We will assume wherever necessary that $f$ is normalized so that its critical point is $c=1 \in S^{1}$. Since $f$ is real-analytic, it extends to a holomorphic map $f: A_{R} \rightarrow \mathbb{C} / \mathbb{Z}$, where $A_{R}$ is the annulus $\{z \in \mathbb{C} / \mathbb{Z}:|\operatorname{Im} z|<R\}$. Making $R$ smaller if necessary, we may assume that $f$ has no critical points outside $S^{1}$. Using Koebe's distortion theorem, it is easy to see that there exists $R_{0}>0$ such that, if $z \in S^{1}$ and $f(z)$ is at a distance $>R_{0}$ from the critical value of $f$, then the inverse branch $f^{-1}$ which maps $f(z)$ back to $z$ is well defined and univalent on the disk $D\left(f(z), R_{0}\right)$. 
On an intuitive level, the key to the proof of Theorem 3.1 is to show that for all sufficiently large $n$ the $n$-th renormalization of $f$ satisfies an inequality of the form $\left|\mathcal{R}^{n}(f)(z)\right| \geq C|z|^{3}$ on a neighborhood of the origin, where $C$ is a universal constant. Thus, the relevant inverse branches of $\mathcal{R}^{n}(f)$ behave as cube roots, mapping a large disk about the origin well within itself, giving rise to a holomorphic pair. The proof depends on the following crucial proposition, which is the analogue of Lemma 3.1 in [15. Following [15], for each $m \geq 1$ we define $D_{m} \subseteq \mathbb{C} / \mathbb{Z}$ to be the disk of diameter the interval $\left[f^{q_{m+1}}(c), f^{q_{m}-q_{m+1}}(c)\right] \subseteq S^{1}$ containing the critical point $c$. Note that diam $\left(D_{m}\right)$ is comparable with $\left|I_{m}\right|$ : this follows from the real a-priori bounds (Theorem 2.1).

Proposition 3.2. There exist universal constants $B_{1}$ and $B_{2}$ and for each $N \geq 1$ there exists $n(N)$ such that for all $n \geq n(N)$ the inverse branch $f^{-q_{n+1}+1}$ taking $f^{q_{n+1}}\left(I_{n}\right)$ back to $f\left(I_{n}\right)$ is well defined and univalent over $\Omega_{n, N}=\left(D_{n-N} \backslash S^{1}\right) \cup$ $f^{q_{n+1}}\left(I_{n}\right)$, and for all $z \in \Omega_{n, N}$ we have

$$
\frac{\operatorname{dist}\left(f^{-q_{n+1}+1}(z), f\left(I_{n}\right)\right)}{\left|f\left(I_{n}\right)\right|} \leq B_{1}\left(\frac{\operatorname{dist}\left(z, I_{n}\right)}{\left|I_{n}\right|}\right)+B_{2} .
$$

Yampolsky's proof of this fact in [15] is greatly facilitated by his assumption that the circle map has the Epstein property (namely, its lift to $\mathbb{C}$ has univalent inverse branches globally defined in the upper half-plane). Thus, the Poincaré neighborhoods $\mathbb{P}_{\theta}(J)$ are automatically invariant in the sense that $f^{-1}\left(\mathbb{P}_{\theta}(J)\right) \subseteq$ $\mathbb{P}_{\theta}\left(f^{-1} J\right)$. In our setting this is no longer true, but as we look at smaller and smaller scales we see that it is asymptotically true, as the following lemma shows.

Lemma 3.3. For every small $a>0$, there exists $\theta(a)>0$ satisfying $\theta(a) \rightarrow 0$ and $a / \theta(a) \rightarrow 0$ as $a \rightarrow 0$, such that the following holds. Let $F: \mathbb{D} \rightarrow \mathbb{C}$ be univalent and symmetric about the real axis, and assume $F(0)=0, F(a)=a$. Then for all $\theta \geq \theta(a)$ we have $F\left(\mathbb{P}_{\theta}([0, a])\right) \subseteq \mathbb{P}_{\left(1-a^{1+\delta}\right) \theta}([0, a])$, where $0<\delta<1$ is an absolute constant.

Proof. There exists a Moebius transformation $G$ such that $G(0)=0, G(a)=a$ and $\left|D^{j} F(x)-D^{j} G(x)\right| \leq C_{0} a^{3-j}$ for $j=0,1,2$ and all $x \in[0, a]$, where $C_{0}$ is an absolute constant (consider the Moebius transformation with the same 2-jet as $F$ at zero, post-composed with a linear map to meet the normalization condition $G(a)=a)$. This $G$ has no pole in a disk $D_{0} \subseteq \mathbb{D}$ of definite radius around zero. Let $\varphi(z)=F(z)-G(z)$. Then $\varphi(z)=b_{1} z+b_{2} z^{2}+\cdots$ for all $z \in D_{0}$, where $\left|b_{1}\right| \leq C_{0} a^{2}$ and $\left|b_{2}\right| \leq C_{0} a$. Take a small number $\varepsilon>0$ and consider the disk $D_{1}=D\left(0, a^{1-\varepsilon}\right) \subseteq D_{0}$. In this disk we have the estimate

$$
|\varphi(z)| \leq\left|b_{1}\right||z|+\left|b_{2}\right||z|^{2}+C_{1}|z|^{3} \leq C_{2} a^{2-2 \varepsilon}|z| .
$$

At the same time, using the fact that $F^{\prime}(\zeta)=1$ for some $\zeta \in[0, a]$ and the Koebe distortion lemma, we see that $|F(z)| \geq C_{3}|z|$ and $|G(z)| \geq C_{3}|z|$ for all $z \in D_{1}$. Therefore, for every such $z$ the triangle with vertices at $0, F(z)$ and $G(z)$ has an angle at zero $\leq C_{4} a^{2-2 \varepsilon}$; similarly for the angle at $a$ in the triangle with vertices $a, F(z)$ and $G(z)$. Now suppose $z \in \mathbb{P}_{\theta}([0, a])$, for $\theta \geq a^{\varepsilon}$. Since $G$ preserves this neighborhood, $G(z)$ forms an angle $\geq \theta$ with $(-\infty, 0]$, and the same holds for the angle $G(z)$ forms with $[a,+\infty)$. It follows that $F(z) \in \mathbb{P}_{\theta^{\prime}}([0, a])$, where $\theta^{\prime}=\theta-C_{4} a^{2-2 \varepsilon} \geq \theta\left(1-a^{1+\delta}\right)$ for some $0<\delta<1$ depending only on $\varepsilon$. This proves the lemma with $\theta(a)=a^{\varepsilon}$. 
The main consequence of this lemma is the following.

Lemma 3.4. For each $n \geq 1$ there exist $K_{n} \geq 1$ and $\theta_{n}>0$, with $K_{n} \rightarrow 1$ and $\theta_{n} \rightarrow 0$ as $n \rightarrow \infty$, such that for all $\theta \geq \theta_{n}$ and all $1 \leq j \leq q_{n+1}$ the inverse branch $f^{-j+1}$ mapping $f^{j}\left(I_{n}\right)$ back to $f\left(I_{n}\right)$ is well defined over $\mathbb{P}_{\theta}\left(f^{j}\left(I_{n}\right)\right)$ and maps this neighborhood univalently into $\mathbb{P}_{\theta / K_{n}}\left(f\left(I_{n}\right)\right)$.

Proof. Let $d_{n}=\max _{1 \leq j \leq q_{n+1}}\left|f^{j}\left(I_{n}\right)\right|$; from the real bounds, these numbers go to zero exponentially with $n$. Take $\delta>0$ as in Lemma 3.3, and let $K_{n}$ be given by

$$
K_{n}^{-1}=\prod_{j=1}^{q_{n+1}}\left(1-\left|f^{j}\left(I_{n}\right)\right|^{1+\delta}\right)
$$

Then define $\theta_{n}=K_{n} \theta\left(d_{n}\right)$, where $\theta(\cdot)$ is the function in Lemma 3.3. Note that

$$
\log K_{n} \leq C \sum_{j=1}^{q_{n+1}}\left|f^{j}\left(I_{n}\right)\right|^{1+\delta} \leq C d_{n}^{\delta}
$$

Therefore $K_{n} \rightarrow 1$ and $\theta_{n} \rightarrow 0$ as required. Also, $d_{n} / \theta_{n} \rightarrow 0$.

Now fix $j$ as in the statement and suppose $\theta \geq \theta_{n}$. Define inductively $\vartheta_{0}=\theta$ and $\vartheta_{i+1}=\left(1-\left|f^{j-i}\left(I_{n}\right)\right|^{1+\delta}\right) \vartheta_{i}$ for $i=0,1, \ldots, j-2$, and note that $\vartheta_{j-1} \geq \theta / K_{n}$. Moreover,

$$
\operatorname{diam}\left(\mathbb{P}_{\vartheta_{i}}\left(f^{j-i}\left(I_{n}\right)\right)\right)=\frac{\left|f^{j-i}\left(I_{n}\right)\right|}{\sin \vartheta_{i}} \leq \frac{C^{\prime} d_{n}}{\theta\left(d_{n}\right)} \ll R_{0} .
$$

Therefore $f^{-1}$ is well defined and univalent over $\mathbb{P}_{\vartheta_{i}}\left(f^{j-i}\left(I_{n}\right)\right)$, and by Lemma 2.4 we have the inclusion $f^{-1}\left(\mathbb{P}_{\vartheta_{i}}\left(f^{j-i}\left(I_{n}\right)\right)\right) \subseteq \mathbb{P}_{\vartheta_{i+1}}\left(f^{j-i-1}\left(I_{n}\right)\right)$. This completes the proof.

Remark. The same result holds true if we replace $I_{n}$ by any interval $J \supseteq I_{n}$ such that the map $f^{q_{n+1}-1}: f(J) \rightarrow f^{q_{n+1}}(J)$ is a diffeomorphism.

We will need four lemmas concerning the sequence $\left\{D_{m}\right\}$ introduced earlier. The first is an easy consequence of Lemma 3.4 and the above remark.

Lemma 3.5. There exists $m_{0} \geq 1$ such that for all $m \geq m_{0}$ the inverse branch $f^{-q_{m}+1}$ taking $f^{q_{m}}\left(I_{m}\right)$ back to $f\left(I_{m}\right)$ is well defined and univalent in $D_{m}$, and

$$
\frac{\operatorname{diam}\left(f^{-q_{m}+1}\left(D_{m}\right)\right)}{\left|I_{m}\right|} \leq C \frac{\operatorname{diam}\left(f\left(D_{m}\right)\right)}{\left|f\left(I_{m}\right)\right|} .
$$

The second is the analogue of Lemma 4.1 in [15].

Lemma 3.6. There exist $\varepsilon_{1}>0$ and $m_{1} \geq m_{0}$ such that for all $m \geq m_{1}$ and each $w \in f^{-q_{m+1}}\left(D_{m}\right) \backslash D_{m}$ we have (a) dist $\left(w, I_{m}\right) \leq C\left|I_{m}\right|$ and (b) for each $x \in I_{m}$, $\varepsilon_{1}<|\arg (w-x)|<\pi-\varepsilon_{1}$.

Proof. The same proof given in [15] applies here. Invariance of Poincaré neighborhoods is replaced by quasi-invariance, using Lemma 3.4.

The third is the analogue of Lemma 4.4 in [15]. It provides us with the tools we need for the inductive step in the proof of Proposition 3.2 (see Figure 2).

Lemma 3.7. There exist $\varepsilon_{2}>0$ and $m_{2} \geq m_{0}$ such that the following holds for all $m \geq m_{2}$. Let $\zeta \in D_{m} \backslash D_{m+1}$ be a point not on the circle, and let $\zeta^{\prime}=f^{-q_{m}}(\zeta)$ and $\zeta^{\prime \prime}=f^{-q_{m+2}}\left(\zeta^{\prime}\right)$. Then either $\zeta^{\prime \prime} \in D_{m+1}$, or else $\operatorname{dist}\left(\zeta^{\prime \prime}, I_{m+1}\right) \leq C\left|I_{m}\right|$ and $\varepsilon_{2}<\arg \left(\zeta^{\prime \prime}-x\right)<\pi-\varepsilon_{2}$ for all $x \in I_{m} \cup I_{m+1}$. 


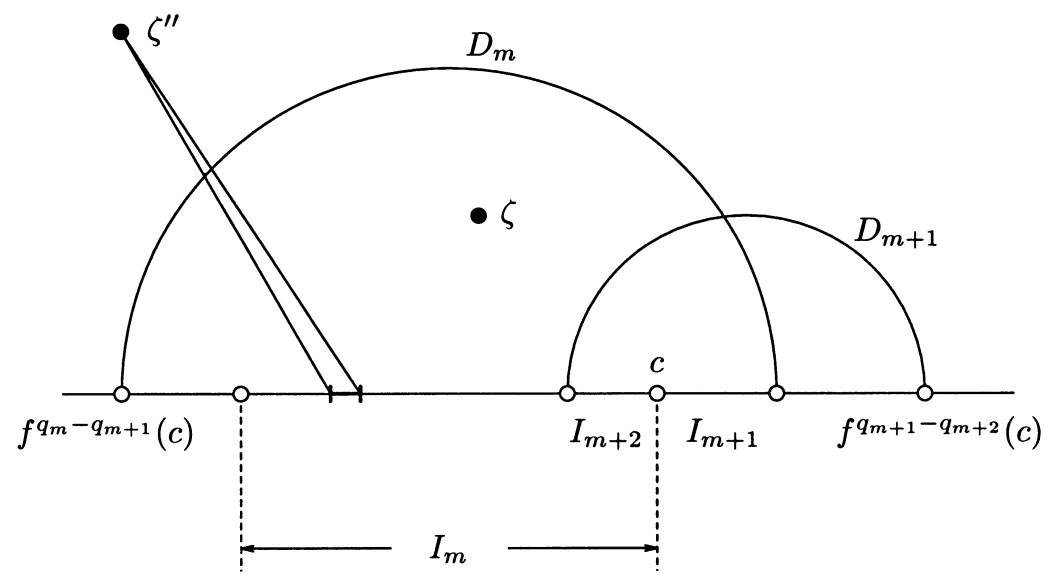

FiguRE 2.

Proof. Once again, the proof of Lemma 4.4 in [15] can be repeated here, mutatis mutandis.

Notation. Given a point $\zeta \in \mathbb{C}$ and an interval $J=(a, b) \subseteq \mathbb{R}$, we denote by angle $(\zeta, J)$ the smallest of the angles $\pi-\arg (\zeta-a)$ and $\arg (\zeta-b)$.

The fourth is a consequence of Lemma 2.5 .

Lemma 3.8. There exist universal constants $N_{*}>0$ and $a_{*}>0$ and some $m_{3}>0$ with the following property. For all $m \geq m_{3}$ such that $a_{m+1}>a_{*}$ and each $w \in$ $V_{m}=f^{-q_{m+1}}\left(D_{m}\right) \backslash D_{m}$, there exists $1<i<N_{*}$ such that the iterate $\left(f^{-q_{m+1}}\right)^{i}(w)$ is well defined and belongs to $D_{m}$.

Proof. Lift $f^{-q_{m+1}}$ to the real line and normalize it so that $I_{m} \backslash I_{m+2}$ becomes the interval $[0,1]$ to get an almost parabolic map $\phi_{m}$. Note that $\phi_{m} \in \mathcal{F}_{\sigma}$ for some $\sigma$ depending only on the real bounds. Let $W_{m}$ be the image of $V_{m}$ under such normalization. It is an easy matter to check that $f^{-q_{m+1}}\left(D_{m}\right) \cap S^{1} \subseteq D_{m} \cap S^{1}$, so that $V_{m}$ does not intersect $S^{1}$, and that $W_{m}^{+}=W_{m} \cap \mathbb{H}$ is compactly contained in $\mathbb{H}$. Therefore, by Lemma 3.6, there exists a fixed compact set $W \subset \mathbb{H}$ such that $W_{m}^{+} \subseteq W$ for all sufficiently large $m$. Similarly, the normalized copies of $D_{m}$ contain a fixed open set $D \supseteq[0,1]$ for all sufficiently large $m$. Hence we can take $N_{*}$ and $a_{*}$ as given by Lemma 2.5 .

Proof of Proposition 3.2. We will start with a point $z$ in the disk $D_{n-N}$. For the argument to work, $n$ will have to be sufficiently large. We start taking $n>N+$ $\max \left\{m_{1}, m_{2}, m_{3}\right\}$, where $m_{1}, m_{2}$ and $m_{3}$ are given respectively by Lemma 3.6, Lemma 3.7 and Lemma 3.8.

In keeping with Yampolsky's notation in [15], let us denote by $J_{-i}$ the interval $f^{q_{n+1}-i}\left(I_{n}\right)$. Also, given $z$, let $z_{-i}=f^{-i}(z)$ be the corresponding pre-images of $z$.

The proof runs by finite induction in the range $n-N \leq m \leq n$. Let $m$ be the largest with the property that $z \in D_{m}$, and keep in mind that dist $\left(z, I_{n}\right) \asymp\left|I_{m}\right|$. Consider those moments $i_{1}<i_{2}<\cdots<i_{\ell}$ in the backward orbit $\left\{J_{-i}\right\}$ before the first return to $I_{m+1}$ such that $J_{-i_{k}} \subseteq I_{m}$. Then, there are two possibilities.

The first possibility is that $z_{-i_{\ell}} \notin D_{m}$. In this case there exists a smallest $k \leq \ell$ such that $z_{-i_{s}} \notin D_{m}$ for $s=k, k+1, \ldots, \ell$. We claim that $\left|J_{-i_{k}}\right| \asymp\left|I_{n}\right|$. This is 
clear from the real bounds if $\ell=a_{m+1} \leq a_{*}$, where $a_{*}$ is given by Lemma 3.8. If on the other hand $\ell>a_{*}$, then again by Lemma 3.8 we must have $\ell-k<N_{*}$, and the claim follows from Lemma 2.2. Therefore, by Lemma 3.6,

$$
\frac{\operatorname{dist}\left(z_{-i_{k}}, J_{-i_{k}}\right)}{\left|J_{-i_{k}}\right|} \leq C \frac{\left|I_{m}\right|}{\left|J_{-i_{k}}\right|} \leq C^{\prime} \frac{\operatorname{dist}\left(z, I_{n}\right)}{\left|I_{n}\right|} .
$$

Moreover, angle $\left(z_{-i_{k}}, J_{-i_{k}}\right) \geq \varepsilon_{1}$, so there exists $\theta=\theta\left(\varepsilon_{1}, N\right)$ such that $z_{-i_{k}} \in$ $\mathbb{P}_{\theta}\left(J_{-i_{k}}\right)$. Now, if $n$ is sufficiently large, $\theta_{n}<\theta$ and we can use Lemma 3.4 to get that $z_{-q_{n+1}+1} \in \mathbb{P}_{\theta / K_{n}}\left(f\left(I_{n}\right)\right)$. This gives us

$$
\frac{\operatorname{dist}\left(z_{-q_{n+1}+1}, f\left(I_{n}\right)\right)}{\left|f\left(I_{n}\right)\right|} \leq C^{\prime \prime} K_{n} \frac{\operatorname{dist}\left(z_{-i_{k}}, J_{-i_{k}}\right)}{\left|J_{-i_{k}}\right|}
$$

and this together with (2) yields the proposition in this case.

The second possibility is that $\zeta=z_{-i_{\ell}} \in D_{m}$, and we can assume that $\zeta \notin D_{m+1}$ (otherwise the induction step is complete). In this case, consider $\zeta^{\prime}=f^{-q_{m}}(\zeta)$ and $\zeta^{\prime \prime}=f^{-q_{m+2}}\left(\zeta^{\prime}\right)$ and the corresponding interval $J^{\prime \prime}=f^{-q_{m}-q_{m+2}}\left(J_{-i_{\ell}}\right)$, and apply Lemma 3.7. Then either $\zeta^{\prime \prime} \in D_{m+1}$, in which case the induction step is complete, or else dist $\left(\zeta^{\prime \prime}, I_{m+1}\right) \leq C\left|I_{m}\right|$ and angle $\left(\zeta^{\prime \prime}, J^{\prime \prime}\right) \geq \varepsilon_{1}$, in which case we can apply the same argument leading to (2) and (3).

If the backward orbit survives all the steps of the induction, this means that in the end $z_{-q_{n+1}+q_{n-1}} \in D_{n-1}$. By Lemma 3.5, the image of $D_{n-1}$ under $f^{-q_{n-1}+1}$ has diameter comparable to $\left|f\left(I_{n}\right)\right|$, so the first member of (3) is simply bounded by an absolute constant. So in any case we have (1).

Proof of Theorem 3.1. First we remark that, since $f$ is a cubic critical circle map, there exists a neighborhood $\Omega$ of the critical point of $f$ such that the restriction $f: \Omega \rightarrow f(\Omega)$ is of the form $f=\psi \circ Q$ where $\psi$ is a univalent map with universally bounded distortion and $Q$ is the map $z \mapsto z^{3}$.

Let $B_{1}, B_{2}$ be the constants of Proposition 3.2 and let us fix a large integer $N$. How large $N$ must be will be determined in the course of the argument. By Proposition 3.2, if $n \geq n(N)$, then inequality (1) holds true for all $z \in \Omega_{n, N}$. Making $n$ larger still if necessary, we have $f^{-q_{n+1}+1}\left(D_{n-N}\right) \subseteq f(\Omega)$. By the above remark, the branch of $f^{-1}$ mapping $f\left(I_{n}\right)$ back to $I_{n}$ is the composition of a cube root with a univalent map with bounded distortion. Using this fact and inequality (1), we have

$$
\frac{\operatorname{diam}\left(f^{-q_{n+1}}\left(D_{n-N}\right)\right)}{\left|I_{n}\right|} \leq C \sqrt[3]{B_{1} \frac{\operatorname{diam}\left(D_{n-N}\right)}{\left|I_{n}\right|}+B_{2}},
$$

for some universal constant $C>0$.

Now, as we know from the real bounds (Theorem 2.1), there exist universal constants $K_{2}>K_{1}>1$ such that, for all sufficiently large $n$,

$$
K_{1}^{N} \leq \frac{\operatorname{diam}\left(D_{n-N}\right)}{\left|I_{n}\right|} \leq K_{2}^{N}
$$

Taking $N \geq 1$ to be the smallest integer greater than $3 \log \left(2 C \sqrt[3]{B_{1}+B_{2}}\right) / 2 \log K_{1}$, we can check from (4) and the first inequality in (5) that

$$
\frac{\operatorname{diam}\left(f^{-q_{n+1}}\left(D_{n-N}\right)\right)}{\left|I_{n}\right|}<\frac{1}{2} \frac{\operatorname{diam}\left(D_{n-N}\right)}{\left|I_{n}\right|} .
$$


Note that $N$ depends only on $B_{1}, B_{2}, K_{1}, C$, and is therefore universal. From these facts, we see at once that for all sufficiently large $n$ the topological disk $f^{-q_{n+1}}\left(D_{n-N}\right)$ is compactly contained in $D_{n-N}$, and moreover

$$
\bmod \left(D_{n-N} \backslash f^{-q_{n+1}}\left(D_{n-N}\right)\right) \geq \mu,
$$

where $\mu>0$ is a universal constant. With these basic geometric bounds at hand, we can easily construct the holomorphic pair to which $\mathcal{R}^{n}(f)$ extends, in the following way. For more details on this construction, see $\S 4$ of [2].

First, let $\hat{f}$ be the standard lift of $f$ to the real line. For each $n$, let $\lambda_{n}=$ $T^{-p_{n}} \circ \hat{f}^{q_{n}}(0)$ and denote by $\Lambda_{n}$ the linear map $x \mapsto \lambda_{n} x$. Take the topological disks $\widetilde{\mathcal{V}}_{n}=D_{n-N}$ and $\widetilde{\mathcal{O}}_{\eta_{n}}=f^{-q_{n+1}}\left(D_{n-N}\right)$ in the cylinder and consider their lifted and normalized copies in $\mathbb{C}$, namely $\mathcal{V}_{n}=\Lambda_{n}^{-1}\left(\exp ^{-1}\left(\widetilde{\mathcal{V}}_{n}\right)\right)$ and $\mathcal{O}_{\eta_{n}}=\Lambda_{n}^{-1}\left(\exp ^{-1}\left(\widetilde{\mathcal{O}}_{\eta_{n}}\right)\right)$ (here, $\exp ^{-1}$ denotes the inverse branch of the exponential that maps the critical point $c=1 \in S^{1}$ of $f$ to the origin). Then consider the map

$$
\eta_{n}=\Lambda_{n}^{-1} \circ T^{-p_{n+1}} \circ \hat{f}^{q_{n+1}} \circ \Lambda_{n}: \mathcal{O}_{\eta_{n}} \rightarrow \mathbb{C} .
$$

The geometric estimates proved above show that $\mathcal{O}_{\eta_{n}}$ is compactly contained in $\mathcal{V}_{n}$, while $\eta_{n}\left(\mathcal{O}_{\eta_{n}}\right) \subseteq \mathcal{V}_{n}$ holds by construction. We define the domains $\mathcal{O}_{\xi_{n}}, \mathcal{O}_{\nu_{n}}$ and the maps $\xi_{n}: \mathcal{O}_{\xi_{n}} \rightarrow \mathbb{C}, \nu_{n}: \mathcal{O}_{\nu_{n}} \rightarrow \mathbb{C}$ in a similar way. We obtain in this fashion a holomorphic pair $\Gamma_{n}$ whose underlying critical commuting pair is precisely the $n$-th renormalization $\mathcal{R}^{n}(f)$. In addition, (6) shows that the conformal modulus of $\Gamma_{n}$ is bounded from below by $\mu$. Finally, it is straightforward to check that all the above topological disks have piecewise analytic boundaries, consisting of finitely many analytic arcs meeting at definite angles, so $\Gamma_{n}$ has geometric boundaries. This completes the proof of the theorem.

Remark. Note from the second inequality in (5) that $\operatorname{diam}\left(\mathcal{V}_{n}\right) \leq K_{2}^{N}$ for all sufficiently large $n$. In other words, the size of the co-domain of $\Gamma_{n}$ is universally bounded from above. This will be very useful later ( $c f$. condition $\mathrm{G}_{2}$ in $\S 4$ below).

\section{GeOmetric CONTROL OF HOLOMORPhiC PAIRS}

In this section, we show that if a holomorphic pair satisfies certain geometric constraints, then one can bound from below how much its dynamics expands the hyperbolic metric in the complement of the real axis. From the renormalization viewpoint, these constraints are quite natural, being a consequence of the complex bounds.

We start with the definition of geometric control. For brevity, let us say that a finite set in the plane is $K$-bounded $(K>1)$, if any two non-zero distances $d_{1}$ and $d_{2}$ between points in the set satisfy $d_{1} \leq K d_{2}$. We denote by $Q$ the map $z \mapsto z^{3}$ in the complex plane.

Definition. A holomorphic pair $\Gamma$ is controlled by $K>1$, or simply $K$-controlled, if the following geometric conditions are satisfied.

$\left(\mathrm{G}_{1}\right)$ The set $\{0, \xi(0), \eta(0), \nu(0), a, b\}$ is $K$-bounded.

$\left(\mathrm{G}_{2}\right)$ We have $\operatorname{diam}(\mathcal{V}) \leq K|J|$.

$\left(\mathrm{G}_{3}\right)$ On the real axis, $\left|\gamma^{\prime}(x)\right| \leq K$ for $\gamma=\xi, \eta, \nu$.

$\left(\mathrm{G}_{4}\right) \xi$ has holomorphic extensions $\xi_{a}: D\left(a, K^{-1}|J|\right) \rightarrow \mathbb{C}$ and $\xi_{0}: D\left(0, K^{-1}|J|\right) \rightarrow$ $\mathbb{C}$ such that $\xi_{a}=\psi_{a} \circ Q \circ \phi_{a}$ and $\xi_{0}=\psi_{\xi} \circ Q$ respectively, where $\psi_{a}, \phi_{a}$ and $\psi_{\xi}$ are univalent maps with distortion bounded by $K$, and $\phi_{a}(a)=0$. 
$\left(\mathrm{G}_{5}\right) \eta$ has holomorphic extensions $\eta_{b}: D\left(b, K^{-1}|J|\right) \rightarrow \mathbb{C}$ and $\eta_{0}: D\left(0, K^{-1}|J|\right) \rightarrow$ $\mathbb{C}$ such that $\eta_{b}=\psi_{b} \circ Q \circ \phi_{b}$ and $\eta_{0}=\psi_{\eta} \circ Q$ respectively, where $\psi_{b}, \phi_{b}$ and $\psi_{\eta}$ are univalent maps with distortion bounded by $K$, and $\phi_{b}(b)=0$.

$\left(\mathrm{G}_{6}\right)$ The open sets $\mathcal{O}_{\xi} \cap D\left(a, K^{-1}|J|\right)$ and $\mathcal{O}_{\eta} \cap D\left(b, K^{-1}|J|\right)$ are connected.

$\left(\mathrm{G}_{7}\right)$ We have $D\left(0, K^{-1}|J|\right) \subseteq \mathcal{O}_{\nu}$.

$\left(\mathrm{G}_{8}\right)$ We have $\bmod (\mathcal{V} \backslash \mathcal{U}) \geq K^{-1}$.

Remark. Condition $\mathrm{G}_{6}$ guarantees that the shadow of a $K$-controlled holomorphic pair extends to a single-valued map

$$
F_{*}: \operatorname{Dom}(\Gamma) \cup D\left(a, K^{-1}|J|\right) \cup D\left(b, K^{-1}|J|\right) \rightarrow \mathbb{C} .
$$

We call this map the extended shadow of our holomorphic pair.

Now a strengthened version of the complex bounds can be stated as follows.

Theorem 4.1. There exists a universal constant $K_{0}>1$ such that the following holds. If $\Gamma$ is a holomorphic pair with arbitrary rotation number, there exists $n_{0}=$ $n_{0}(\Gamma)$ such that for all $n \geq n_{0}$ the holomorphic pair $\mathcal{R}^{n} \Gamma$ restricts to a holomorphic pair $\Gamma_{n}$ controlled by $K_{0}$.

Proof. This is straightforward from the real bounds given by Theorem 2.1 and the complex bounds given by Theorem 3.1. For condition $\mathrm{G}_{2}$, use the remark following the proof of Theorem 3.1. For conditions $\mathrm{G}_{4}$ and $\mathrm{G}_{5}$, use also Koebe distortion.

Let us now formulate the tools from function theory that we need to prove expansion of the hyperbolic metric by a controlled holomorphic pair. Given a hyperbolic Riemann surface $X$, we write $\rho_{X}$ for the hyperbolic density of $X, d_{X}$ for the hyperbolic metric of $X$, and $\operatorname{diam}_{X}(E)$ for the diameter of $E \subseteq X$ in that metric. If $f: \mathcal{O} \subseteq X \rightarrow Y$ is a holomorphic map between two hyperbolic Riemann surfaces $(\mathcal{O}$ an open subset of $X)$, we denote by

$$
\left\|f^{\prime}(z)\right\|_{X, Y}=\frac{\rho_{Y}(f(z))}{\rho_{X}(z)}\left|f^{\prime}(z)\right|
$$

the derivative of $f$ at $z \in X$ measured with respect to the hyperbolic metrics in $X$ and $Y$, and simplify the notation a bit to $\left\|f^{\prime}(z)\right\|_{Y}$ when $X=Y$.

The key to expansion is the following lemma, stated as Prop. 4.9 in [10].

Lemma 4.2. There exists a universal positive function $C(s)$ decreasing to zero as $s$ decreases to zero such that the following holds. If $f: X \subset Y$ is the strict inclusion of a hyperbolic Riemann surface into another, then we have the inequality $\left\|f^{\prime}(z)\right\|_{X, Y} \leq C\left(d_{Y}(z, Y \backslash f(X))\right)<1$.

The second tool we need, to be used in combination with the above, is the following.

Lemma 4.3. Let $\Omega$ be a doubly-connected region in the plane, let $E$ be the bounded component of $\mathbb{C} \backslash \Omega$, and let $V=E \cup \Omega$. Then we have

$$
\operatorname{diam}_{V}(E) \leq \frac{C}{\bmod (\Omega)},
$$

for some constant $C>0$, as well as

$$
\bmod (\Omega) \geq \frac{4}{\pi}\left[\frac{\delta}{\operatorname{diam}(\Omega)}\right]^{2}
$$

where $\delta$ is the Euclidean distance between the boundary components of $\Omega$. 
Proof. For (7), see [9], Th. 2.4, where in fact a more precise estimate is given . For $(8)$, use the definition of $\bmod (\Omega)$ as the extremal length of the family $\mathcal{F}$ of curves in $\Omega$ joining the two components of $\partial \Omega$ to get

$$
\bmod (\Omega) \geq \frac{1}{A(\Omega)} \inf _{\gamma \in \mathcal{F}} L^{2}(\gamma),
$$

where $L(\gamma)$ is the Euclidean length of $\gamma$ and $A(\Omega)$ is the Euclidean area of $\Omega$. Since $A(\Omega) \leq \pi(\operatorname{diam} \Omega)^{2} / 4$, whereas $L(\gamma) \geq \delta$ for all $\gamma \in \mathcal{F}$, this proves $(8)$.

While inequality (8) gives a lower bound for the modulus of an annulus in terms of the minimum separation between boundary components, an upper bound is provided by Teichmüller's inequality

$$
\bmod (\Omega) \leq \Phi\left(\frac{\delta}{\operatorname{diam} \Omega}\right),
$$

where $\Phi(x)$ is a certain universal function, which is continuous and monotone increasing, and which tends to zero as $x \rightarrow 0 ; c f$. [1].

Later in this section it will also be very important to be able to control the variation of holomorphic distortion of an analytic map between hyperbolic Riemann surfaces. When the time comes, we will resort to the following consequence of Koebe's distortion theorem, as stated by McMullen in [9], Cor. 2.27.

Lemma 4.4. Let $f: X \rightarrow Y$ be an analytic map between hyperbolic Riemann surfaces whose derivative vanishes nowhere. Then for all $x_{1}, x_{2} \in X$ we have

$$
\left\|f^{\prime}\left(x_{1}\right)\right\|_{X, Y}^{\alpha} \leq\left\|f^{\prime}\left(x_{2}\right)\right\|_{X, Y} \leq\left\|f^{\prime}\left(x_{1}\right)\right\|_{X, Y}^{1 / \alpha},
$$

where $\alpha=\exp \left\{C_{0} d_{X}\left(x_{1}, x_{2}\right)\right\}$ and $C_{0}>0$ is a universal constant.

Besides these very general tools, we will need the following specific consequence of geometric control. Given $E \subseteq \mathbb{C}$ and $\varepsilon>0$, we write $V(E, \varepsilon)=\{z: d(z, E)<$ $\varepsilon \operatorname{diam}(E)\}$.

Lemma 4.5. Given $K>1$, there exist $\varepsilon_{0}>0$ and $M>1$ such that the following holds for all $0<\varepsilon \leq \varepsilon_{0}$. If $\Gamma$ is a $K$-controlled holomorphic pair and $F_{*}$ is its extended shadow, then

(a) $V(J, \varepsilon) \subseteq \operatorname{Dom}\left(F_{*}\right)$;

(b) for all $z \in V(J, \varepsilon)$ we have $\left|F_{*}^{\prime}(z)\right| \leq M$;

(c) there exists $0<\tau=\tau(\varepsilon, K)<\varepsilon$ such that $F_{*}(V(J, \tau)) \subseteq V\left(F_{*}(J), \varepsilon\right)$;

(d) $F_{*}(V(J, \varepsilon)) \subseteq \operatorname{Dom}(\Gamma)$.

Proof. Everything boils down to controlling the map $F_{*}$ on a neighborhood of $J$. Let $\Delta=F_{*}(J)=[\xi(a), \eta(b)]=[\xi(a), \xi(0)]$. To prove (a), we note that

$$
\bmod (\mathcal{V} \backslash \Delta)>\bmod (\mathcal{V} \backslash \operatorname{Dom}(\Gamma)) \geq K^{-1}
$$

by condition $\mathrm{G}_{8}$. Hence, there exists $\varepsilon_{1}>0$ depending only on $K$ such that $V\left(\Delta, \varepsilon_{1}\right) \subseteq \mathcal{V}$, by Teichmüller's inequality (9). Using condition $\mathrm{G}_{4}$ and making $\varepsilon_{1}$ smaller if necessary, we find $0<\varepsilon_{2}<K^{-1}$ and $0<\varepsilon_{3}<\varepsilon_{1}$, both depending only on $K$, such that

$$
\left\{\begin{array}{l}
D\left(\xi(a), \varepsilon_{3}|\Delta|\right) \subseteq \xi_{a}\left(D\left(a, \varepsilon_{2}|J|\right)\right) \subseteq V\left(\Delta, \varepsilon_{1}\right) \\
D\left(\xi(0), \varepsilon_{3}|\Delta|\right) \subseteq \xi_{0}\left(D\left(0, \varepsilon_{2}|J|\right)\right) \subseteq V\left(\Delta, \varepsilon_{1}\right)
\end{array}\right.
$$


Now, consider $a<x<0$. If $|\xi(x)-\xi(a)|<\varepsilon_{3}|\Delta|$, then by (10) we have $x \in D\left(a, \varepsilon_{2}|J|\right)$. Similarly, if $|\xi(x)-\xi(0)|<\varepsilon_{3}|\Delta|$, then $x \in D\left(0, \varepsilon_{2}|J|\right)$. If neither of these happen, then

$$
\xi(a)+\varepsilon_{3}|\Delta|<\xi(x)<\xi(0)-\varepsilon_{3}|\Delta| .
$$

In this case the disk $D_{x}=D\left(\xi(x), \varepsilon_{3}|\Delta|\right) \subseteq V\left(\Delta, \varepsilon_{1}\right)$ is contained in the image of $\xi$. Since $\left|\xi^{\prime}(x)\right| \leq K$ by condition $\mathrm{G}_{3}$, and since $|\Delta| \geq K^{-1}|J|$ by condition $\mathrm{G}_{1}$, we deduce from Koebe's one-quarter theorem that

$$
\xi^{-1}\left(D_{x}\right) \supseteq D\left(x, \frac{\varepsilon_{3}|J|}{4 K^{2}}\right) .
$$

Putting these facts together, and denoting the extended $\xi$ by $\xi_{*}$, we get

$$
\xi_{*}^{-1}\left(V\left(\Delta, \varepsilon_{1}\right)\right) \supseteq V\left([a, 0], \varepsilon^{\prime}\right),
$$

where $\varepsilon^{\prime}=\min \left\{\varepsilon_{2}, \varepsilon_{3} / 4 K^{2}\right\}$. Proceeding similarly with $\eta$ replacing $\xi$ and condition $\mathrm{G}_{5}$ replacing condition $\mathrm{G}_{4}$, and noting that $\Delta \supseteq[\eta(0), \eta(b)]$, we obtain $\varepsilon^{\prime \prime}>0$ depending only on $K$ such that

$$
\eta_{*}^{-1}\left(V\left(\Delta, \varepsilon_{1}\right)\right) \supseteq V\left([0, b], \varepsilon^{\prime \prime}\right),
$$

where $\eta_{*}$ is the extension of $\eta$. Taking $\varepsilon_{0}=\min \left\{|a| \varepsilon^{\prime},|b| \varepsilon^{\prime \prime}\right\} /|J|$, we deduce from (11) and (12) that $V\left(J, \varepsilon_{0}\right) \subseteq \operatorname{Dom}\left(F_{*}\right)$, and this proves (a).

The proof of the remaining assertions is more of the same, so we omit the details. Using condition $\mathrm{G}_{3}$ and Koebe distortion, we see that the maps defining $F_{*}$ have distortion on $V\left(J, \varepsilon_{0} / 2\right)$ bounded only in terms of $K$. Therefore (b) holds for some constant $M$ depending only on $K$ if we replace $\varepsilon_{0}$ by $\varepsilon_{0} / 2$. Making this new $\varepsilon_{0}$ still smaller if necessary, we can assume also that $V\left(\Delta, \varepsilon_{0}\right) \subseteq \operatorname{Dom}(\Gamma)$. Then, (c) follows for some $\tau$ as stated if we use (b) and apply the mean value theorem to $F_{*}$ in $V(J, \tau)$. Finally, replacing $\varepsilon_{0}$ by $\tau\left(\varepsilon_{0}, K\right)$, we see that (d) follows from (c).

Next, let $\Gamma$ be a holomorphic pair and let $Y=\mathcal{V} \backslash \mathbb{R}$. How do we compare the hyperbolic metric of $Y$ with the hyperbolic metric of the upper half-plane $\mathbb{H}$ ? The answer is given by the following lemma.

Lemma 4.6. Let $\rho_{Y}(z)$ and $\rho(z)=1 /|\operatorname{Im} z|$ be the hyperbolic densities of $Y$ and $\mathbb{C} \backslash \mathbb{R}$, respectively. Then for all $z \in \operatorname{Dom}(\Gamma)$ we have $\rho(z)<\rho_{Y}(z) \leq c_{M} \rho(z)$, where $c_{M}>1$ is a constant depending only on $M=\bmod (\mathcal{V} \backslash \operatorname{Dom}(\Gamma))$.

Proof. By monotonicity of hyperbolic densities, $\rho_{Y}>\rho$. Let $\delta$ be the Euclidean distance between $\partial \mathcal{V}$ and $\partial \operatorname{Dom}(\Gamma)$. By Teichmüller's inequality (9), we have

$$
\delta \geq \Phi^{-1}(M) \operatorname{diam} \mathcal{V} \geq \Phi^{-1}(M)|\operatorname{Im} z| .
$$

Hence the largest disk $D$ contained in $Y$ and centered at $z$ has radius

$$
R \geq \min \{\delta,|\operatorname{Im} z|\} \geq 2 c_{M}^{-1}|\operatorname{Im} z|,
$$

where $c_{M}^{-1}=\frac{1}{2} \min \left\{1, \Phi^{-1}(M)\right\}$. Therefore, again by monotonicity of hyperbolic densities, we get

$$
\rho_{Y}(z) \leq \rho_{D}(z)=\frac{2}{R} \leq c_{M} \frac{1}{|\operatorname{Im} z|}=c_{M} \rho(z),
$$

and this completes the proof. 
We are ready for the first expansion result.

Proposition 4.7. Let $\Gamma$ be a $K$-controlled holomorphic pair with co-domain $\mathcal{V}$, and let $Y=\mathcal{V} \backslash \mathbb{R}$. Then for each $\varepsilon>0$ we have the following.

(a) There exists $\varrho=\varrho(\varepsilon, K)>0$ satisfying $\left\|F^{\prime}(z)\right\|_{Y} \geq 1+\varrho$ for all $z$ in the domain of $\Gamma$ such that $|\operatorname{Im} z| \geq \varepsilon|J|$.

(b) There exists $\lambda=\lambda(\varepsilon, K)>1$ such that $\left\|F^{\prime}(z)\right\|_{Y} \geq \lambda$ for all $z \in \operatorname{Dom}(\Gamma) \cap$ $V(J, \varepsilon)$ such that $F(z) \notin V(J, \varepsilon)$.

Moreover, if $\Gamma$ is the renormalization of a holomorphic pair $\Gamma^{*}$ with co-domain $\mathcal{V}^{*}$ and $Y^{*}=\mathcal{V}^{*} \backslash \mathbb{R}$, then the same statements are true with $\|\cdot\|_{Y^{*}}$ replacing $\|\cdot\|_{Y}$ throughout.

Proof. It suffices to consider only $\varepsilon \leq \varepsilon_{0}$, where $\varepsilon_{0}$ is given by Lemma 4.5. By condition $\mathrm{G}_{8}$ and (3), there exists $\varepsilon_{1}=\varepsilon_{1}(K)>0$ such that the Euclidean distance between $\partial \operatorname{Dom}(\Gamma)$ and $\partial \mathcal{V}$ is at least $\varepsilon_{1}|J|$.

Let $E$ be the set of points in $\operatorname{Dom}(\Gamma)^{+} \subseteq \mathcal{V}^{+}$such that $\operatorname{Im} z \geq \varepsilon|J|$, and let $\delta$ be the Euclidean distance between the boundary components of the annulus $\mathcal{V}^{+} \backslash E$. Then we have

$$
\delta \geq|J| \min \left\{\varepsilon_{1}, \varepsilon\right\}
$$

Moreover, by condition $\mathrm{G}_{2}$,

$$
\operatorname{diam}\left(\mathcal{V}^{+} \backslash E\right) \leq \operatorname{diam}(\mathcal{V}) \leq K|J| .
$$

Combining (13) and (14) with inequalities (7) and (8) of Lemma 4.3, we get an upper bound for the diameter of $E$ in the hyperbolic metric of $\mathcal{V}^{+}$, namely

$$
\operatorname{diam}_{\mathcal{V}^{+}}(E) \leq \frac{C_{0} \pi K^{2}}{4\left[\min \left\{\varepsilon_{1}, \varepsilon\right\}\right]^{2}}=s .
$$

Now let $z \in E$ and suppose that $F(z)=\xi(z)$, i.e. that $z \in \mathcal{O}_{\xi}^{+}$(other cases being treated in the same way). If $w$ is any point in the non-empty set $\partial \mathcal{O}_{\xi}^{+} \cap \partial E$, we have

$$
d_{\mathcal{V}^{+}}\left(z, \mathcal{V}^{+} \backslash \mathcal{O}_{\xi}^{+}\right) \leq d_{\mathcal{V}^{+}}(z, w) \leq \operatorname{diam}_{\mathcal{V}^{+}}(E)
$$

Viewed as a (univalent) map from $\mathcal{V}^{+}$into itself, $\xi^{-1}$ is the composition of the hyperbolic isometry $\xi^{-1}: \mathcal{V}^{+} \rightarrow \mathcal{O}_{\xi}^{+}$with the hyperbolic contraction given by the inclusion $\mathcal{O}_{\xi}^{+} \subseteq \mathcal{V}^{+}$. Therefore, by Lemma 4.2 and (16),

$$
\left\|\left(\xi^{-1}\right)^{\prime}(\xi(z))\right\|_{Y} \leq C\left(\operatorname{diam}_{\mathcal{V}^{+}}(E)\right)<1,
$$

which put together with (15) gives us

$$
\left\|F^{\prime}(z)\right\|_{Y}=\left\|\xi^{\prime}(z)\right\|_{Y} \geq \frac{1}{C(s)}>1 .
$$

This proves part (a) with $\varrho(\varepsilon, K)=C(s)^{-1}-1>0$.

To prove part (b) we note, using parts (c) and (d) of Lemma 4.5, that if $z \in$ $\operatorname{Dom}(\Gamma) \cap V(J, \varepsilon)$ is such that $F(z) \notin V(J, \varepsilon)$, then $z \notin V(J, \tau(\varepsilon, K))$. In particular, $\operatorname{Im} z \geq \tau(\varepsilon, K)|J|$, and therefore by (a) we have

$$
\left\|F^{\prime}(z)\right\|_{Y} \geq 1+\varrho(\tau(\varepsilon, K), K)=\lambda(\varepsilon, K) .
$$

This completes the proof of both assertions for $\|\cdot\|_{Y}$. The proof for $\|\cdot\|_{Y^{*}}$ is similar. 
We now turn to our second expansion result.

Proposition 4.8. Let $\Gamma$ be a $K$-controlled holomorphic pair with arbitrary rotation number. Then for each $\mu>1$ there exists $n_{*}=n_{*}(\mu, \Gamma)>0$ with the following property. If $z$ is a point in the domain of $\Gamma$ such that $F^{j}(z) \in \operatorname{Dom}(\Gamma)$ for all $0 \leq j<n$ but $F^{n}(z) \notin \operatorname{Dom}(\Gamma)$ for some $n>n_{*}$, then $\left\|\left(F^{n}\right)^{\prime}(z)\right\|_{Y} \geq \mu$.

Proof. Let $\Delta=[\xi(0), \eta(0)]$ be the short dynamical interval of $\Gamma$, and let $f: \Delta \rightarrow \Delta$ be the associated circle map. Also, let $K_{0}$ and $n_{0}=n_{0}(\Gamma)$ be given by Theorem 4.1. Then for all $n \geq n_{0}$ the $n$-th renormalization of $\Gamma$ is a holomorphic pair with the same co-domain $\mathcal{V}$ that restricts to a $K_{0}$-controlled holomorphic pair $\Gamma_{n}$. We denote by $\mathcal{U}_{n}=\operatorname{Dom}\left(\Gamma_{n}\right)$ the domain and $\mathcal{V}_{n}$ the co-domain of $\Gamma_{n}$, by $F_{n}$ its shadow, by $J_{n}$ its long dynamical interval and by $I_{n}$ its short dynamical interval. We also consider the protection bands $\mathcal{W}_{n}=V\left(J_{n}, \varepsilon_{0}\right)$, where $\varepsilon_{0}$ is the constant in Lemma 4.5. Recall that $F_{n}\left(\mathcal{W}_{n}\right) \subseteq \operatorname{Dom}\left(\Gamma_{n}\right)$.

Since $\left|J_{n}\right| \rightarrow 0$ as $n \rightarrow \infty$, and since diam $\mathcal{V}_{n} \leq K_{0}\left|J_{n}\right|$ by condition $\mathrm{G}_{2}$, there exists a sequence $n_{0}<n_{1}<n_{2}<\cdots<n_{j}<\cdots$ along which domains and co-domains are nested in the sense that

$$
\mathcal{V}_{n_{j+1}} \subseteq \mathcal{U}_{n_{j}} \cap \mathcal{W}_{n_{j}}
$$

holds true for all $j \geq 1$. Now choose an integer $k$ such that $\lambda^{k-1}>\mu$, where $\lambda=\lambda\left(\varepsilon_{0}, K_{0}\right)$ is given by Proposition $4.7(\mathrm{~b})$.

Claim. If $z \in \mathcal{U}_{n_{k}}$ and $n>0$ are such that $F^{j}(z) \in \operatorname{Dom}(\Gamma)$ for $j=0,1, \ldots, n-1$ but $F^{n}(z) \notin \operatorname{Dom}(\Gamma)$, then $\left\|\left(F^{n}\right)^{\prime}(z)\right\|_{Y}>\mu$.

To prove this claim, we proceed as follows. Since $z \in \mathcal{U}_{n_{k}}$ and the $F$-orbit of $z$ eventually exits the domain of $\Gamma$, there exists $s_{k} \geq 1$ such that $F_{n_{k}}^{i}(z) \in \mathcal{U}_{n_{k}}$ for all $0 \leq i<s_{k}$ but $z_{k-1}=F_{n_{k}}^{s_{k}}(z) \notin \mathcal{U}_{n_{k}}$. By the nesting condition (17), we must have $z_{k-1} \in \mathcal{U}_{n_{k-1}} \cap \mathcal{W}_{n_{k-1}}$. Proceeding inductively in this fashion until the $F$-orbit of $z$ exits $\mathcal{U}_{n_{1}}$, we obtain positive integers $s_{k}, s_{k-1}, \ldots, s_{1}$ and points $z_{k}=z, z_{k-1}, \ldots, z_{0}$ satisfying

$$
z_{j-1}=F_{n_{j}}^{s_{j}}\left(z_{j}\right) \in \mathcal{U}_{n_{j-1}} \cap \mathcal{W}_{n_{j-1}}
$$

and such that $F_{n_{j}}^{i}\left(z_{j}\right) \in \mathcal{U}_{n_{j}}$ for all $0 \leq i<s_{j}$ while $z_{j-1} \notin \mathcal{U}_{n_{j}}$, for all $1 \leq j \leq k$. Note that since the $F$-orbit of $z$ exits each $\mathcal{U}_{n_{j}}$, it exits each protection band $\mathcal{W}_{n_{j}}$ a fortiori. Therefore, by Proposition 4.7 (b), we have $\left\|\left(F_{n_{j}}^{s_{j}}\right)^{\prime}\left(z_{j}\right)\right\|_{Y} \geq \lambda$ for $j=1,2, \ldots, k-1$. When the $F$-orbit of $z$ reaches $z_{0}$, there are still, say, $r$ iterates to go before it finally exits the domain of $\Gamma$, and we see that

$$
F^{n}(z)=F^{r} \circ F_{n_{1}}^{s_{1}} \circ F_{n_{2}}^{s_{2}} \circ \cdots \circ F_{n_{k}}^{s_{k}}(z) .
$$

Therefore by the chain rule we have

$$
\left\|\left(F^{n}\right)^{\prime}(z)\right\|_{Y}=\left\|\left(F^{r}\right)^{\prime}\left(z_{0}\right)\right\|_{Y} \prod_{j=1}^{k}\left\|\left(F_{n_{j}}^{s_{j}}\right)^{\prime}\left(z_{j}\right)\right\|_{Y}>\lambda^{k-1}>\mu,
$$

which proves the claim.

The next step ( $c f$. Figure 3 ) is to cover $J$ with pre-images of $\mathcal{U}_{n_{k}}$ under $F$. More precisely, since $\mathcal{U}_{n_{k}} \supseteq I_{n_{k}}$ and

$$
\Delta=\bigcup_{j=0}^{q_{n_{k}}} f^{-j}\left(I_{n_{k}}\right)
$$




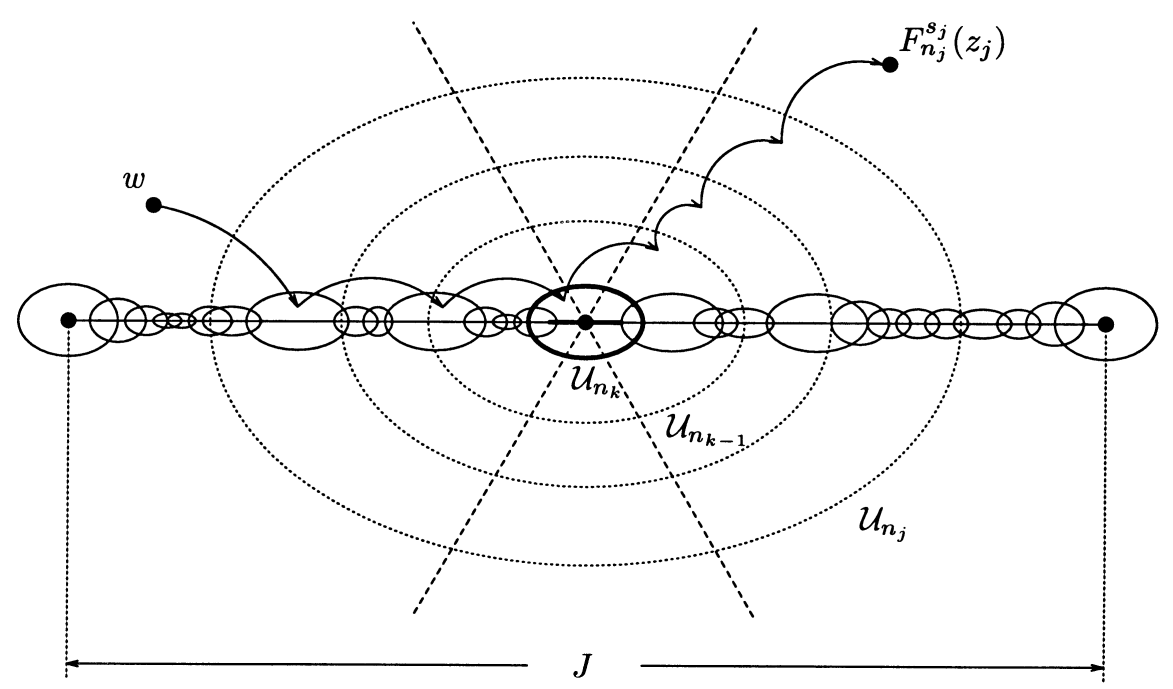

FiguRE 3.

it follows that $\Delta$ is contained in the open set

$$
\mathcal{O}=\bigcup_{j=0}^{q_{n_{k}}} F^{-j}\left(\mathcal{U}_{n_{k}}\right) .
$$

Here we have used the fact that $f$ is simply the restriction of $F$ to the short dynamical interval of $\Gamma$. Now recall that $J=\Delta \cup \eta^{-1}(\Delta) \cup \xi^{-1}(\Delta) \cup \cdots \cup \xi^{-m}(\Delta)$, where $m$ is the height of $\Gamma$. Hence $J$ is contained in the open set

$$
\mathcal{O}^{\prime}=\bigcup_{j=0}^{m} F_{*}^{-j}(\mathcal{O})
$$

where $F_{*}$ is the extended shadow of $\Gamma$ (we use $F_{*}$ instead of $F$ so we can cover the endpoints of $J$ also). This means that for some $\varepsilon>0$ small enough $V(J, \varepsilon) \subseteq \mathcal{O}^{\prime}$. All points in the domain of $\Gamma$ that are not in $V(J, \varepsilon)$ lie at a definite positive distance from the real axis, in other words,

$$
\varepsilon^{\prime}=\inf \{|\operatorname{Im} z|: z \in \operatorname{Dom}(\Gamma) \backslash V(J, \varepsilon)\}>0 .
$$

Hence, let $n_{*}$ be such that $(1+\varrho)^{n_{*}}>\mu$, where $\varrho=\varrho\left(\varepsilon^{\prime}, K\right)$ is given by Proposition 4.7 (a). If $w \in \operatorname{Dom}(\Gamma)$ and $n>n_{*}$ are such that $F^{j}(w) \in \operatorname{Dom}(\Gamma)$ for all $0 \leq j<n$ but $F^{n}(w) \notin \operatorname{Dom}(\Gamma)$, then either there exists $j$ such that $F^{j}(w) \in V(J, \varepsilon)$, in which case there exists $i \geq j$ such that $z=F^{i}(w) \in \mathcal{U}_{n_{k}}$ and therefore by the Claim above

$$
\left\|\left(F^{n}\right)^{\prime}(w)\right\|_{Y} \geq\left\|\left(F^{n-i}\right)^{\prime}(z)\right\|_{Y} \geq \mu
$$

or $F^{j}(w) \notin V(J, \varepsilon)$ for all $j<n$, in which case $\left|\operatorname{Im} F^{j}(w)\right| \geq \varepsilon^{\prime}|J|$, whence by Proposition 4.7 (a) and the chain rule we get $\left\|\left(F^{n}\right)^{\prime}(w)\right\|_{Y} \geq(1+\varrho)^{n}>\mu$ also. This completes the proof.

In order to prove our next result about holomorphic pairs, we shall need the following property of maps in the Arnold family. 
Lemma 4.9. Let $f: \mathbb{C}^{*} \rightarrow \mathbb{C}^{*}$ be a map in the Arnold family. If the rotation number of $f \mid \partial \mathbb{D}$ is irrational, then the Julia set of $f$ is $\mathbb{C}^{*}$.

Proof. A well-known generalization of Sullivan's no-wandering domains theorem states that a holomorphic self-map of $\mathbb{C}^{*}$ with finitely many singular values has no wandering domains (see [7] for a proof). The lemma follows from this and the fact that $f$ has no non-repelling cycles or Herman rings, since its unique critical point has an orbit dense in the unit circle.

Definition. A generalized holomorphic pair is a conformal copy of a holomorphic pair, in other words, the conjugate of a holomorphic pair by a univalent map. The image of a bowtie under a univalent map is called a generalized bowtie.

Proposition 4.10. Let $\Gamma$ be a holomorphic pair with arbitrary rotation number, and let $\mathcal{K}_{\Gamma}$ be its limit set. Then

$$
\mathcal{K}_{\Gamma}=\overline{\bigcup_{n \geq 0} F^{-n}(J)} .
$$

Proof. Since the statement we want to prove is purely topological, we can assume, using the pull-back theorem for holomorphic pairs (Theorem 2.3) and Theorem 4.1, that $\Gamma$ satisfies all the hypotheses of Proposition 4.8. For the same reasons, we also know that $\Gamma$ is conjugate to a deep renormalization of a suitable element $f$ of the Arnold family. More precisely, there exist domains $\mathcal{V}^{0}, \mathcal{O}_{\xi}^{0}, \mathcal{O}_{\eta}^{0}, \mathcal{O}_{\nu}^{0} \subseteq \mathbb{C}^{*}$, symmetric about $\partial \mathbb{D}$ with respect to inversion, forming a (generalized) bowtie with center at $1 \in \partial \mathbb{D}$, and a positive integer $s$ such that the maps

$$
\xi_{0}=f^{q_{s}}\left|\mathcal{O}_{\xi}^{0}, \eta_{0}=f^{q_{s+1}}\right| \mathcal{O}_{\eta}^{0}, \nu_{0}=f^{q_{s}+q_{s+1}} \mid \mathcal{O}_{\nu}^{0}
$$

determine a generalized holomorphic pair $\Gamma^{0}$, and moreover there exists a quasiconformal homeomorphism $H: \mathcal{V} \rightarrow \mathcal{V}^{0}$ conjugating $\Gamma$ to $\Gamma^{0}$. Let $F_{0}$ be the shadow of $\Gamma^{0}$, and let $J_{0}=H(J) \in \partial \mathbb{D}$ be the long dynamical interval of $\Gamma^{0}$. The distinctive feature of $\Gamma^{0}$ is the fact that, for each $w \in \operatorname{Dom}\left(\Gamma^{0}\right)$ and each $k>0$ for which $F_{0}^{k}(w)$ is defined, there exist non-negative integers $m, n$ with $k \leq m+n$ such that

$$
F_{0}^{k}(w)=f^{m q+n Q}(w),
$$

where $q=q_{s}$ and $Q=q_{s+1}$.

Claim. The set $\Lambda=\mathcal{K}_{\Gamma} \backslash \bigcup_{n \geq 0} F^{-n}(J)$ has empty interior.

To prove this, we argue by contradiction. Suppose $D \subseteq \Lambda$ is a non-empty open disk, and let $D_{0}=H(D)$. Since points in $D$ can be iterated by $F$ forever, $F_{0}^{k}(w)$ exists for all $k \geq 0$, for each $w \in D_{0}$, and so by (18) there exist infinitely many $n>0$ such that $f^{n}(w) \in \operatorname{Dom}\left(\Gamma_{0}\right)$. From Lemma 4.9, we know that the Julia set of $f$ is $\mathbb{C}^{*}$, so by Montel's theorem there exist $n_{0}>0$ and $w \in D_{0}$ such that $f^{n_{0}}(w) \in \partial \mathbb{D}$. But then $f^{n}(w) \in \partial \mathbb{D}$ for all $n \geq n_{0}$, and so there exists $k>0$ such that $F_{0}^{k}(w) \in \partial \mathbb{D} \cap \operatorname{Dom}\left(\Gamma_{0}\right)=J_{0}$. We have thus found a point $z=H^{-1}(w)$ in $D$ which belongs to $F^{-k}(J)$, a contradiction that proves the claim.

Now, let $x$ be an arbitrary point of $\Lambda$. We want to show that for every $\delta>0$ there exists a point $p \in D(x, \delta)$ such that $F^{m}(p) \in J$ for some $m>0$. Since $F^{n}(x)$ exists for all $n \geq 0$ and lies in the interior of $\operatorname{Dom}(\Gamma)$, it follows from the claim above that for all $\delta>0$ and all sufficiently large $k$ there exists $y \in D(x, \delta)$ such that $F^{j}(y) \in \operatorname{Dom}(\Gamma)$ for $0 \leq j<k$ but $F^{k}(y) \notin \operatorname{Dom}(\Gamma)$. By an easy 
connectedness argument we can assume also that $F^{k}(y) \in \partial \operatorname{Dom}(\Gamma)$. We call such $y$ a $(k, \delta)$-escaping point for $x$.

Next, observe that $C_{1}=\sup \left\{d_{Y}\left(z, F^{-1}(J) \cap Y\right): z \in \partial \operatorname{Dom}(\Gamma)\right\}<\infty$. Given $\delta>0$, let $\mu>1$ be such that

$$
\mu>\left(\frac{2 C_{1}}{\delta}\right)^{e^{C_{0} C_{1}}}
$$

where $C_{0}$ is the universal constant of Lemma 4.4. Let $y$ be an $(n, \delta / 2)$-escaping point for $x$ with $n>n_{*}$, where $n_{*}$ is given by Proposition 4.8, and let $w=F^{n}(y) \in$ $\partial \operatorname{Dom}(\Gamma)$. Take any point $w^{*} \in F^{-1}(J)$ such that $d_{Y}\left(w, w^{*}\right) \leq C_{1}$. Denote by $F^{-n}$ the inverse branch of $F^{n}$ that maps $w$ back to $y$, and let $p=F^{-n}\left(w^{*}\right)$.

To estimate the Euclidean distance between $x$ and $p$, we first estimate the hyperbolic distance in $Y$ between $y$ and $p$. For this purpose, let $\sigma$ be the hyperbolic geodesic in $Y$ joining $w$ to $w^{*}$, and let $\rho_{Y}$ be the hyperbolic density of $Y$. The distance $d_{Y}(y, p)$ is certainly not greater than the hyperbolic length $L_{Y}\left(F^{-n} \sigma\right)$ of the $\operatorname{arc} F^{-n} \sigma$ joining $y$ to $p$. On the other hand, by Proposition 4.8 we have $\left\|\left(F^{-n}\right)^{\prime}(w)\right\|_{Y} \leq \mu^{-1}$, and applying Lemma 4.4 we get

$$
\left\|\left(F^{-n}\right)^{\prime}(z)\right\|_{Y} \leq \frac{1}{\mu^{1 / \alpha}},
$$

for all $z \in \sigma$, where $\alpha=\exp \left\{C_{0} d_{Y}(w, z)\right\} \leq e^{C_{0} C_{1}}$. Hence, using (19) and (20), we see that

$$
\begin{aligned}
L_{Y}\left(F^{-n} \sigma\right) & =\int_{\sigma}\left\|\left(F^{-n}\right)^{\prime}(z)\right\|_{Y} \rho_{Y}(z)|d z| \\
& \leq \frac{1}{\mu^{1 / \alpha}} \int_{\sigma} \rho_{Y}(z)|d z|=\frac{1}{\mu^{1 / \alpha}} d_{Y}\left(w, w^{*}\right) \\
& \leq C_{1} \mu^{-e^{-C_{0} C_{1}}}<\frac{\delta}{2} .
\end{aligned}
$$

This gives us $|y-p| \leq d_{Y}(y, p)<\delta / 2$, and therefore $|x-p|<\delta$ as desired.

The main consequence of Proposition 4.10 is one of the key points we shall use in $\S 6$ to prove that the limit set of a holomorphic pair is uniformly twisting. Let $z$ be a point in the limit set $\mathcal{K}_{\Gamma}$ of a holomorphic pair $\Gamma$. For each tangent vector $v$ at $z$, we write $\ell_{Y}(v)$ for the length of $v$ measured in the hyperbolic metric of $Y$, and put $\ell_{Y}(v)=\infty$ if $z$ happens to lie in the real axis. For each $k \geq 0$, we let $v_{k}=D F^{k}(z) v$.

Theorem 4.11. Let $\Gamma$ be a holomorphic pair with arbitrary rotation number, and let $z$ be a point in the limit set $\mathcal{K}_{\Gamma}$. Then, for each tangent vector $v$ at $z$, we have

$$
\ell_{Y}\left(v_{0}\right) \leq \ell_{Y}\left(v_{1}\right) \leq \cdots \leq \ell_{Y}\left(v_{k}\right) \rightarrow \infty
$$

as $k \rightarrow \infty$.

Proof. The sequence is clearly non-decreasing, so we concentrate on proving that it diverges to infinity. Assume $z \in \mathcal{K}_{\Gamma} \cap Y$, otherwise there is nothing to prove, and fix $k>0$. Let $\mathcal{O}_{k} \subseteq \operatorname{Dom}(\Gamma)$ be the connected component of $F^{-k}(Y)$ that contains $z$. We know that $F^{k}$ maps $\mathcal{O}_{k}$ univalently onto one of the two connected components of $Y$. For definiteness, let us assume that $\mathcal{O}_{k} \subseteq \mathcal{V}^{+}$and that $F^{k}\left(\mathcal{O}_{k}\right)=\mathcal{V}^{+}$. Thus, 
viewed as a map from $\mathcal{V}^{+}$into itself, $F^{-k}$ is the composition of an isometry between $\mathcal{V}^{+}$and $\mathcal{O}_{k}$, with their respective hyperbolic metrics, and a contraction given by the inclusion $\mathcal{O}_{k} \subseteq \mathcal{V}^{+}$. In particular,

$$
\left\|D F^{-k}\left(F^{k}(z)\right)\right\|_{Y}=\frac{\rho_{Y}(z)}{\rho_{\mathcal{O}_{k}}(z)} .
$$

Now, if $D_{k}$ is the largest disk centered at $z$ and contained in $\mathcal{O}_{k}$, then

$$
\rho_{\mathcal{O}_{k}}(z) \asymp \rho_{D_{k}}(z)=\frac{2}{R_{k}},
$$

where $R_{k}$ is the radius of $D_{k}$. We also saw in Lemma 4.6 that $\rho_{Y}(z) \asymp 1 /|\operatorname{Im} z|$. Combining these facts with (21) and the chain rule, we get

$$
\ell_{Y}\left(v_{k}\right)=\left\|\left(F^{k}\right)^{\prime}(z)\right\|_{Y} \ell_{Y}(v) \asymp \frac{2|\operatorname{Im} z|}{R_{k}} \ell_{Y}(v) .
$$

Since by Proposition 4.10 we have $R_{k} \rightarrow 0$ as $k \rightarrow \infty$, we are done.

\section{The CRITICAL POINT IS DEEP}

Now we use the expansion results established in the previous section to prove that the critical point of a holomorphic pair $\Gamma$ is a $\delta$-deep point of the limit set $\mathcal{K}_{\Gamma}$ of $\Gamma$, for some $\delta>0$.

Definition. Let $\Lambda \subseteq \mathbb{C}$ be compact. We say that a point $p \in \Lambda$ is a $\delta$-deep point of $\Lambda$ if for every $r>0$ the largest disk contained in $D(p, r)$ which does not intersect $\Lambda$ has radius $\leq r^{1+\delta}$.

In particular, if $p$ is $\delta$-deep for $\Lambda$, then linear blow-ups of $\Lambda$ around $p$ fill-out the plane at an exponential rate in the Hausdorff metric.

Theorem 5.1. Let $\Gamma$ be a holomorphic pair with arbitrary rotation number and limit set $\mathcal{K}_{\Gamma}$. Then there exists $\delta>0$ such that the critical point of $\Gamma$ is a $\delta$-deep point of $\mathcal{K}_{\Gamma}$.

Proof. In the course of the proof, we write $C_{0}$ for the constant of Lemma 4.4, and $C_{1}, C_{2}, \ldots$ for constants that depend only on the complex bounds. Let $\Gamma_{n}$ be the $n$-th renormalization of $\Gamma$. By Theorem 4.1 we may assume that $\Gamma_{n}$ is $K_{0}$-controlled for all $n \geq n_{0}$. As in the proof of Proposition 4.8, we choose $n_{0}<n_{1}<\cdots n_{i}<\cdots$ so that $\mathcal{V}_{n_{i+1}} \subseteq \mathcal{U}_{n_{i}} \cap \mathcal{W}_{n_{i}}$, where the $\mathcal{W}_{n}$ are the protection bands defined in that proposition. Since $\operatorname{diam}\left(\mathcal{U}_{n}\right) \asymp \operatorname{diam}\left(\mathcal{V}_{n}\right) \asymp\left|J_{n}\right|$ and $\left|J_{n}\right| \rightarrow 0$ exponentially as $n \rightarrow \infty$, we can choose the $n_{i}$ so that $n_{i+1}-n_{i}$ is bounded (by a constant depending only on the real bounds). This entails $n_{i} \leq C_{1} i$ for all $i$.

We want to show that for every $z$ in the domain of $\Gamma$ such that $z \notin \mathcal{K}_{\Gamma}$ we have $\operatorname{dist}\left(z, \mathcal{K}_{\Gamma}\right) \leq C_{2}|z|^{1+\delta}$ for some $\delta>0$. Let $k$ be the largest integer such that $z \in \mathcal{U}_{n_{k}}$. Then $\operatorname{diam}\left(\mathcal{U}_{n_{k}}\right) \asymp|z|$, and by the real bounds

$$
n_{k} \asymp \log \left(\frac{1}{\left|J_{n_{k}}\right|}\right) \asymp \log \left(\frac{1}{|z|}\right) \text {. }
$$

Since $n_{k} \leq C_{1} k$, we get $k \geq C_{3} \log (1 /|z|)$. Now, since $z \notin \mathcal{K}_{\Gamma}$, its forward orbit eventually leaves $\mathcal{U}$, and so it leaves each $\mathcal{U}_{n_{i}}$, and each $\mathcal{W}_{n_{i}}$ as well. Let $n$ be such 
that $F^{n}(z) \in \mathcal{U}_{n_{0}} \cap \mathcal{W}_{n_{0}}$ but $F^{n+1}(z) \notin \mathcal{W}_{n_{0}}$. Then, by the same argument used in the proof of the Claim in Proposition 4.8, we have

$$
\left\|D F^{n}(z)\right\|_{Y} \geq \lambda^{k} \geq\left(\frac{1}{|z|}\right)^{C_{3} \log \lambda}=\frac{1}{|z|^{\beta}} .
$$

Moreover, by Lemma 4.5 (c), the point $z^{\prime}=F^{n}(z)$ satisfies $\operatorname{Im} z^{\prime} \geq \tau\left(\varepsilon_{0}, K_{0}\right)\left|J_{n_{0}}\right|$, and so we can find a point $w^{\prime} \in \mathcal{K}_{\Gamma} \cap \partial \mathcal{W}_{n_{0}}$ such that $d_{Y}\left(z^{\prime}, w^{\prime}\right) \leq C_{4}$. Let $\gamma^{\prime}$ be the geodesic arc in $Y$ joining $z^{\prime}$ to $w^{\prime}$ (so that $\left.L_{Y}\left(\gamma^{\prime}\right) \leq C_{4}\right)$. Then the arc $\gamma=F^{-n}\left(\gamma^{\prime}\right)$ joins $z$ to $w=F^{-n}\left(w^{\prime}\right) \in \mathcal{K}_{\Gamma}$. Using Lemma 4.4 , we see that $\left\|D F^{-n}(\zeta)\right\|_{Y} \leq$ $\left\|D F^{-n}(z)\right\|_{Y}^{\alpha}$ for all $\zeta \in \gamma^{\prime}$, where $\alpha=e^{C_{0} C_{4}}$. Therefore $L_{Y}(\gamma) \leq C_{5}|z|^{\delta}$, where $\delta=\alpha \beta$. Since the hyperbolic density of $Y$ at points in $\mathcal{U}$ is comparable to the hyperbolic density of the upper half-plane $\mathbb{H}$, it follows that $L_{\mathbb{H}}(\gamma) \leq C_{6}|z|^{\delta}$. Thus, $w$ belongs to the hyperbolic disk $\Omega \subseteq \mathbb{H}$ of center $z$ and radius $C_{6}|z|^{\delta}$. Since the point of $\partial \Omega$ farthest from $z$ in the Euclidean metric lies vertically above $z$, let us say $z+i L$, we get

$$
\log \left(1+\frac{L}{\operatorname{Im} z}\right) \leq C_{6}|z|^{\delta}
$$

But since $|z|$ is bounded, the left-hand side of this inequality is $\geq C_{7} L / \operatorname{Im} z$, and we finally have

$$
|w-z| \leq L \leq \frac{C_{6}}{C_{7}}|z|^{\delta}(\operatorname{Im} z) \leq C_{8}|z|^{1+\delta} .
$$

We deduce that the largest Euclidean disk centered at $z \notin \mathcal{K}_{\Gamma}$ which does not meet $\mathcal{K}_{\Gamma}$ has radius $\leq C_{8}|z|^{1+\delta}$, and this proves the theorem.

\section{SMAll Limit SETS EVERYWHERE}

In this section we use Theorem 4.11 to prove an important property of holomorphic pairs that will imply $(\S 7)$ that the limit set is uniformly twisting in the sense of McMullen [10]. The precise statement is given in Theorem 6.3 below. We will need the following property of critical circle maps.

Lemma 6.1. Let $f: S^{1} \rightarrow S^{1}$ be a critical circle map for which the real a-priori bounds hold true. For each $n \geq 1$, let $\Delta_{n}$ be the interval of endpoints $f^{q_{n}}(c)$ and $f^{q_{n-1}}(c)$ containing the critical point $c$. Then $\Delta_{n}, f^{-1}\left(\Delta_{n}\right), \ldots, f^{-q_{n}+1}\left(\Delta_{n}\right)$ are pairwise disjoint and each $f^{-j}\left(\Delta_{n}\right)$ has definite and bounded space on both sides inside the largest interval containing it that does not meet any of the others.

Proof. The endpoints of $f^{-j}\left(\Delta_{n}\right)$ are $f^{q_{n}-j}(c)$ and $f^{q_{n+1}-j}(c)$, and both belong to the dynamical partition $\mathcal{P}_{n}$ of level $n$ of $f$.

We will also need an easy property of controlled holomorphic pairs.

Lemma 6.2. Let $\Gamma$ be a controlled holomorphic pair. Then there exist a disk $D^{\prime} \subseteq$ $\mathcal{O}_{\nu} \backslash \mathbb{R}$ and a disk $D^{\prime \prime} \subseteq \mathcal{U}$ centered at the origin, with $\operatorname{diam}\left(D^{\prime}\right) \asymp|J| \asymp \operatorname{diam}\left(D^{\prime \prime}\right)$ and $\operatorname{dist}\left(D^{\prime}, \mathbb{R}\right) \asymp|J|$, such that $\nu$ is univalent in $D^{\prime}$ and $\nu\left(D^{\prime}\right) \supseteq D^{\prime \prime}$.

Proof. Control implies the existence of a disk $D \subseteq \mathcal{U}$ of definite radius around 0 and at a definite distance from $\nu(0)$. In any one of the two components of $\nu^{-1}(D)$ disjoint from $\mathbb{R}$ we find, by Koebe distortion, a disk $D^{\prime}$ of definite radius around the corresponding pre-image of 0 . To obtain $D^{\prime \prime}$ inside $\nu\left(D^{\prime}\right)$, apply Koebe again! 
Definition. We say that a holomorphic pair $\Gamma$ is super-controlled if $\Gamma$ is $K$ controlled and there exist $R=R(K)>0$, domains $\widetilde{\mathcal{O}}_{\xi}, \widetilde{\mathcal{O}}_{\eta}, \widetilde{\mathcal{O}}_{\nu}$, and complexanalytic extensions $\widetilde{\xi}: \widetilde{\mathcal{O}}_{\xi} \rightarrow \mathbb{C}, \widetilde{\eta}: \widetilde{\mathcal{O}}_{\eta} \rightarrow \mathbb{C}, \widetilde{\nu}: \widetilde{\mathcal{O}}_{\nu} \rightarrow \mathbb{C}$ of $\xi, \eta, \nu$ respectively, such that for $\gamma=\xi, \eta, \nu$ and for all $z \in \partial \mathcal{O}_{\gamma} \cap \mathcal{K}_{\Gamma}$ we have $D(z, R|J|) \subseteq \widetilde{\mathcal{O}}_{\gamma}$ and the restriction of $\widetilde{\gamma}$ to $D(z, R|J|)$ has distortion bounded by $K$.

To go from control to super-control, one renormalization suffices. In other words, if $\Gamma$ is $K$-controlled, then its first renormalization $\mathcal{R}(\Gamma)$ is a super-controlled holomorphic pair.

Theorem 6.3. Let $\Gamma$ be a super-controlled holomorphic pair with rotation number of bounded type, with limit set $\mathcal{K}_{\Gamma}$ and shadow $F$. Then for each $z_{0} \in \mathcal{K}_{\Gamma}$ and each $r>0$ there exists a pointed domain $(U, y)$ with $\left|z_{0}-y\right| \asymp r$ and $\operatorname{diam}(U) \asymp r$, and there exists $k>0$ such that $F^{k}$ maps $(U, y)$ onto a pointed domain $(V, 0)$ univalently with bounded distortion. In particular, $U$ contains a conformal copy of some renormalization of $\Gamma$ whose limit set has size commensurable with $r$.

Proof. The proof is divided into several steps.

Step I. First we show that the statement is true when $z_{0} \in[\xi(0), \eta(0)]$. Let $f=$ $F \mid[\xi(0), \eta(0)]$ and for each $n \geq 1$ let $\Delta_{n}$ be as in Lemma 6.1. Each $\Delta_{n}$ is the short dynamical interval of the $n$-th renormalization $\Gamma_{n}$ of $\Gamma$. Let $\mathcal{V}_{n} \supseteq \Delta_{n}$ be the codomain of $\Gamma_{n}$; by the complex bounds, we can assume that $\operatorname{diam}\left(\mathcal{V}_{n}\right) \asymp\left|\Delta_{n}\right|$. For each $0<i \leq q_{n}-1$, let $V_{n, i}=f^{-i}\left(\mathcal{V}_{n}\right)$; note that by Koebe's distortion theorem we have $\operatorname{diam}\left(V_{n, i}\right) \asymp\left|f^{-i}\left(\Delta_{n}\right)\right|$. Now, choose $n$ so that the interval of $\mathcal{P}_{n}$ which contains $z_{0}$ has length $\asymp r$. Then choose $k$ so that $V_{n, k}$ is closest to $z_{0}$. This value of $k$ and the pointed domain $(U, y)=\left(V_{n, k}, f^{-k}(0)\right)$ will do.

Step II. Next, the statement is true for all $z_{0} \in(a, b)$, the long dynamical interval of $\Gamma$. Here, let $p$ be the height of $\Gamma$ and consider the domains $V_{n, i}$ together with $\xi^{-1}\left(V_{n, i}\right), \xi^{-2}\left(V_{n, i}\right), \ldots, \xi^{-p}\left(V_{n, i}\right)$ and $\eta^{-1}\left(V_{n, i}\right)$. Then apply the same argument as in Step I.

Step III. Now we prove the more difficult case when $z_{0}$ is not on the real axis. Here is where we use Theorem 4.11. This case breaks down into further sub-cases. We start with a vector $v_{0}$ at $z_{0}$ and Euclidean norm $\left|v_{0}\right| \asymp r$, and we iterate: $z_{k}=F^{k}(z), v_{k}=D F^{k}(z) v$. Note that $\left|F^{\prime}\left(z_{k}\right)\right|=\left|v_{k+1}\right| /\left|v_{k}\right|$ for all $k$. We also know from Theorem 4.11 that $\ell_{Y}\left(v_{k}\right) \rightarrow \infty$ monotonically as $k \rightarrow \infty$. There are two possibilities.

Step IIIa. There exists $k$ such that $\ell_{Y}\left(v_{k}\right) \ll 1$ but $\ell_{Y}\left(v_{k+1}\right) \gg 1$. More precisely, $\ell_{Y}\left(v_{k}\right)<\varepsilon$ while $\ell_{Y}\left(v_{k+1}\right)>1 / \varepsilon$, for some constant $\varepsilon$ that will be determined in the course of the argument. To be definite, assume $z_{k}$ lies in the domain of $\xi$, so $z_{k+1}=\xi\left(z_{k}\right)$. Since at all points $z$ of the domain of $\Gamma$ the hyperbolic density of $Y$ is comparable to $1 / \operatorname{Im} z$, we have

$$
\frac{\left|v_{k}\right|}{\operatorname{Im} z_{k}} \asymp \ell_{Y}\left(v_{k}\right)<\varepsilon .
$$

Hence $\operatorname{Im} z_{k} \geq C\left|v_{k}\right| / \varepsilon \gg\left|v_{k}\right|$. This means that $\xi$ is univalent in a disk $D\left(z_{k}, R\right)$ of radius $R \asymp \operatorname{Im} z_{k}$ (provided it is well defined there - it may happen that $z_{k}$ is too close to the boundary of the domain of $\xi$, in which case we must remember that $\xi$ extends holomorphically to a definite neighborhood of such boundary because the holomorphic pair $\Gamma$ is super-controlled). By Koebe's one-quarter theorem, 
$\xi\left(D\left(z_{k}, R\right)\right)$ contains the disk $D\left(z_{k+1}, R^{\prime}\right)$, where $R^{\prime}=R\left|v_{k+1}\right| / 4\left|v_{k}\right| \gg\left|v_{k+1}\right|$. But $\ell_{Y}\left(v_{k+1}\right) \asymp\left|v_{k+1}\right| / \operatorname{Im} z_{k+1}$ is large $(>1 / \varepsilon)$, so $\left|v_{k+1}\right| \gg \operatorname{Im} z_{k+1}$. Let $\zeta=\operatorname{Re} z_{k+1}$ be the point on the real axis closest to $z_{k+1}$. By Step II, there exists a pointed domain $\left(U^{\prime \prime}, y^{\prime \prime}\right)$, which is mapped univalently by some iterate of $F$ to a pointed domain $(V, 0)$ around the critical point, such that $\operatorname{diam}\left(U^{\prime \prime}\right) \asymp\left|v_{k+1}\right|$ and $\left|\zeta-y^{\prime \prime}\right|=O\left(\left|v_{k+1}\right|\right)$, so $\left|z_{k+1}-y^{\prime \prime}\right|=O\left(\left|v_{k+1}\right|\right)$ also. Now, if $\varepsilon$ is chosen small enough, we have $U^{\prime \prime} \subseteq D\left(z_{k+1}, R^{\prime} / 2\right)$. Take $U^{\prime}=\xi^{-1}\left(U^{\prime \prime}\right) \subseteq D\left(z_{k}, R\right)$ and $y^{\prime}-\xi^{-1}\left(y^{\prime \prime}\right) \in U^{\prime}$. Again by Koebe's distortion theorem we have $\operatorname{diam}\left(U^{\prime}\right) \asymp\left|v_{k}\right|$ and $\left|z_{k}-y^{\prime}\right|=O\left(\left|v_{k}\right|\right)$. Since $D\left(z_{k}, R\right) \subseteq Y$, the inverse branch $F^{-k}$ mapping $z_{k}$ back to $z_{0}$ is well defined and univalent in $D\left(z_{k}, R\right)$. Thus, take $U=F^{-k}\left(U^{\prime}\right)$ and $y=F^{-k}\left(y^{\prime}\right)$. Once again by Koebe's distortion theorem, $\operatorname{diam}(U) \asymp\left|v_{0}\right| \asymp r$, and $\left|y-z_{0}\right|=O\left(\left|v_{0}\right|\right)=O(|r|)$, so we are done in this case.

Step IIIb. There exists $k$ such that $\ell_{Y}\left(v_{k}\right) \asymp 1$. As in McMullen's original argument, this case is the most delicate. Here, we have $\left|v_{k}\right| \asymp \operatorname{Im} z_{k}$. As before, let $\zeta=\operatorname{Re} z_{k}$ be the point on the real axis closest to $z_{k}$. Using Step II, we find some $V_{n, i}$ and $y_{n, i} \in V_{n, i}$ such that $\operatorname{diam}\left(V_{n, i}\right) \asymp \operatorname{dist}\left(\zeta, y_{n, i}\right) \asymp \operatorname{Im} z_{k}$ (note that it is here that we use the bounded type assumption) and such that $F^{i}$ maps $\left(V_{n, i}, y_{n, i}\right)$ univalently onto $\left(\mathcal{V}_{n}, 0\right)$. Applying Lemma 6.2 to the controlled holomorphic pair $\Gamma_{n}$, we get a disk $D^{\prime}$. Let $U^{\prime}=F^{-i}\left(D^{\prime}\right) \subseteq V_{n, i}$. Using Koebe's distortion theorem, we see that $\operatorname{diam}\left(U^{\prime}\right) \asymp \operatorname{dist}\left(U^{\prime}, \mathbb{R}\right) \asymp \operatorname{diam}\left(V_{n, i}\right)$. It follows at once that if $\gamma$ is the geodesic arc in $Y$ joining $z_{k}$ to $U^{\prime}$, then $\operatorname{diam}\left(\gamma \cup U^{\prime}\right) \asymp \operatorname{Im} z_{k}$. Hence we can find $r_{k} \asymp \operatorname{Im} z_{k}$ and points $\zeta_{1}=z_{k}, \zeta_{2}, \ldots, \zeta_{s} \in \gamma \cup U^{\prime}$ such that

$$
\gamma \cup U^{\prime} \subseteq \Omega=\bigcup_{j=1}^{s} D\left(\zeta_{j}, r_{k} / 2\right),
$$

and such that $D\left(\zeta_{j}, r_{k}\right) \subseteq \mathcal{U} \backslash \mathbb{R}$ for all $j$. But now the inverse branch $\Phi=$ $F^{-k}$ mapping $z_{k}$ back to $z_{0}$ is well defined over the union of the disks $D\left(\zeta_{j}, r_{k}\right)$. Applying Koebe's distortion to each of these disks we see that for all $z \in \Omega$ we have $\left|\Phi^{\prime}(z)\right| \asymp\left|\Phi^{\prime}\left(z_{k}\right)\right| \asymp\left|v_{0}\right| /\left|v_{k}\right|$. But then we see that $U=\Phi\left(U^{\prime}\right)$ satisfies $\operatorname{diam}(U) \asymp\left|v_{0}\right|=r$.

\section{Proof of Theorem 1.1}

The proof of Theorem 1.1 will combine the results of the previous sections with McMullen's powerful dynamic inflexibility theorem for holomorphic dynamical systems. We recall very briefly the relevant definitions and then the statement of McMullen's theorem.

Let us denote by $\mathcal{V}(\widehat{\mathbb{C}} \times \widehat{\mathbb{C}})$ the set of all analytic hypersurfaces of $\widehat{\mathbb{C}} \times \widehat{\mathbb{C}}$. For us, a holomorphic dynamical system is a subset $\mathcal{F} \subseteq \mathcal{V}(\widehat{\mathbb{C}} \times \widehat{\mathbb{C}})$. The elements of $\mathcal{F}$ are its holomorphic relations. A topology in $\mathcal{V}(\widehat{\mathbb{C}} \times \widehat{\mathbb{C}})$ can be defined as follows. If $F \subseteq \widehat{\mathbb{C}} \times \widehat{\mathbb{C}}$ is a hypersurface, its boundary $\partial F=\bar{F} \backslash F$ is closed in $\widehat{\mathbb{C}} \times \widehat{\mathbb{C}}$. Hence, given $F \in \mathcal{V}(\widehat{\mathbb{C}} \times \widehat{\mathbb{C}})$ and a sequence $F_{i} \in \mathcal{V}(\widehat{\mathbb{C}} \times \widehat{\mathbb{C}})$, declare $F_{i} \rightarrow F$ if

(a) $\partial F_{i} \rightarrow \partial F$ in the Hausdorff metric on closed subsets of $\widehat{\mathbb{C}} \times \widehat{\mathbb{C}}$;

(b) for each open set $U \subseteq \widehat{\mathbb{C}} \times \widehat{\mathbb{C}}$ there exist $f, f_{i}: U \rightarrow \mathbb{C}$ (for all sufficiently large $i$ ) such that $U \cap F=f^{-1}(0), U \cap F_{i}=f_{i}^{-1}(0)$, each $f, f_{i}$ vanishes to order one on $F, F_{i}$ respectively, and the sequence $f_{i}$ converges uniformly to $f$ on compact subsets of $U$. 
Then declare a set to be closed in $\mathcal{V}(\widehat{\mathbb{C}} \times \widehat{\mathbb{C}})$ if it contains the limits of all its convergent sequences. As McMullen shows in Ch. 9 of $[10]$, the space $\mathcal{V}(\widehat{\mathbb{C}} \times \widehat{\mathbb{C}})$ with this topology is separable and metrizable.

The geometric topology on the space of all closed holomorphic dynamical systems is by definition the Hausdorff topology on the space of closed subsets of $\mathcal{V}(\widehat{\mathbb{C}} \times \widehat{\mathbb{C}})$. The geometric topology is typically non-Hausdorff (thus non-metrizable), but nevertheless it is sequentially compact.

We may now formulate the following notions, after McMullen [10].

Saturation. Given a holomorphic dynamical system $\mathcal{F}$, we define its saturation $\mathcal{F}^{\text {sat }}$ to be the closure in $\mathcal{V}(\widehat{\mathbb{C}} \times \widehat{\mathbb{C}})$ of the set whose elements are the intersections $F \cap U$, where $F \in \mathcal{F}$ and $U \subseteq \widehat{\mathbb{C}} \times \widehat{\mathbb{C}}$ is open.

Non-linearity. A holomorphic dynamical system $\mathcal{F} \subseteq \mathcal{V}(\widehat{\mathbb{C}} \times \widehat{\mathbb{C}})$ is said to be nonlinear if it does not leave invariant a parabolic line field in $\widehat{\mathbb{C}}$.

Twisting. A (closed) holomorphic dynamical system $\mathcal{F} \subseteq \mathcal{V}(\widehat{\mathbb{C}} \times \widehat{\mathbb{C}})$ is said to be twisting if every holomorphic dynamical system quasiconformally conjugate to $\mathcal{F}$ is non-linear.

Uniform twisting. A family $\left\{\mathcal{F}_{\alpha}\right\}$ of holomorphic dynamical systems is said to be uniformly twisting if every geometric limit of the family of saturations $\left\{\mathcal{F}_{\alpha}^{\text {sat }}\right\}$ is a twisting dynamical system.

The family $(\mathcal{F}, \Lambda)$. Given $\mathcal{F} \subseteq \mathcal{V}(\widehat{\mathbb{C}} \times \widehat{\mathbb{C}})$ and a compact set $\Lambda$ in the Riemann sphere, we define a family $(\mathcal{F}, \Lambda)$ of holomorphic dynamical systems in the following way. For each baseframe $\omega$ in the convex-hull $\operatorname{ch}(\Lambda)$ of $\Lambda$ in hyperbolic 3-space, let $T_{\omega}$ be the fractional linear transformation that sends $\omega$ onto the standard baseframe $\omega_{0}$ at $(0,1) \in \mathbb{C} \times \mathbb{R}_{+} \equiv \mathbb{H}^{3}$. Define $(\mathcal{F}, \omega)$ to be the dynamical system $T_{\omega}^{*}(\mathcal{F})$, the pull-back of $\mathcal{F}$ by $T_{\omega}$. Then let $(\mathcal{F}, \Lambda)$ be the family of all $(\mathcal{F}, \omega)$ as $\omega$ ranges through the baseframes in $\operatorname{ch}(\Lambda)$.

$C^{1+\alpha}$-conformality. A map $\phi: \widehat{\mathbb{C}} \rightarrow \widehat{\mathbb{C}}$ is said to be $C^{1+\alpha}$-conformal at $p \in \widehat{\mathbb{C}}$ (for some $\alpha>0)$ if the complex derivative $\phi^{\prime}(p)$ exists and we have

$$
\phi(z)=\phi(p)+\phi^{\prime}(p)(z-p)+O\left(|z-p|^{1+\alpha}\right)
$$

for all $z$ near $p$.

With these notions at hand, we are now in a position to state McMullen's fundamental dynamic inflexibility theorem. For a proof, see [10], page 166.

Theorem 7.1. Let $\mathcal{F} \subseteq \mathcal{V}(\widehat{\mathbb{C}} \times \widehat{\mathbb{C}})$ be a holomorphic dynamical system, and let $\Lambda \subseteq \widehat{\mathbb{C}}$ be a compact set. If $(\mathcal{F}, \Lambda)$ is uniformly twisting and $\phi: \widehat{\mathbb{C}} \rightarrow \widehat{\mathbb{C}}$ is a Kquasiconformal conjugacy between $\mathcal{F}$ and another holomorphic dynamical system $\mathcal{F}^{\prime}$, then for each $\delta$-deep point $p \in \Lambda$ the map $\phi$ is $C^{1+\alpha}$-conformal at $p$, for some $\alpha>0$.

Remark. The number $\alpha$ depends only on the positive numbers $K, \delta$ and on a very specific measure of the non-linearity of $\mathcal{F}$ defined by McMullen in Ch. 9 of [10]. 
To be able to apply McMullen's theorem, we need to show that our case falls within his framework. The definition of holomorphic dynamical system adopted by McMullen is sufficiently broad to include our case. Indeed, we can define the full dynamics of a holomorphic pair $\Gamma$ as follows.

Intuitively, the full dynamics of a holomorphic pair $\Gamma$ is the class of all holomorphic maps whose graphs are contained in the holomorphic relations of $\Gamma$. These holomorphic relations are defined using the maps $\xi: \mathcal{O}_{\xi} \rightarrow \mathbb{C}, \eta: \mathcal{O}_{\eta} \rightarrow \mathbb{C}$, $\nu: \mathcal{O}_{\nu} \rightarrow \mathbb{C}$ in their open domains. Let $\Omega$ be the set of all finite words in the alphabet $\{\xi, \eta, \nu\}$. Given $w \in \Omega$, let $\operatorname{Dom}(w)$ be the set of all $z \in \operatorname{Dom}(\Gamma)$ such that $w(z)$ exists. Then each $\operatorname{Dom}(w) \subseteq \mathbb{C}$ is an open set. If $\operatorname{Dom}(w)$ is non-empty, we say that $w$ is admissible for $\Gamma$, and the set of all such words is denoted by $\Omega_{\Gamma}$. Now, for each pair $\left(w_{1}, w_{2}\right) \in \Omega_{\Gamma} \times \Omega_{\Gamma}$, let

$$
G\left(w_{1}, w_{2}\right)=\left\{\left(z_{1}, z_{2}\right) \in \operatorname{Dom}\left(w_{1}\right) \times \operatorname{Dom}\left(w_{2}\right): w_{1}\left(z_{1}\right)=w_{2}\left(z_{2}\right)\right\} \subseteq \widehat{\mathbb{C}} \times \widehat{\mathbb{C}} .
$$

Each $G\left(w_{1}, w_{2}\right)$ is an analytic hypersurface in $\widehat{\mathbb{C}} \times \widehat{\mathbb{C}}$. Now consider the set $\mathcal{F}(\Gamma)$ of all holomorphic maps $g: U \rightarrow \mathbb{C}$ for which there exists $\left(w_{1}, w_{2}\right) \in \Omega_{\Gamma} \times \Omega_{\Gamma}$ such that $\operatorname{gr}(g)=\{(z, g(z)): z \in U\} \subseteq G\left(w_{1}, w_{2}\right)$. This set is the full dynamics of $\Gamma$. Due to the presence of the critical point, $\mathcal{F}(\Gamma)$ is certainly non-linear.

Lemma 7.2. Let $\Gamma$ be a super-controlled holomorphic pair with rotation number of bounded type, and let $\mathcal{K}_{\Gamma}$ be its limit set. Then $\left(\mathcal{F}(\Gamma), \mathcal{K}_{\Gamma}\right)$ is uniformly twisting.

Proof. Using Theorem 6.3 , we prove that $\mathcal{F}(\Gamma)$ is uniformly twisting by the same argument as in the proof of Theorem 9.18 in [10.

Let $\omega_{n}$ be a sequence of baseframes in the convex-hull $\operatorname{ch}\left(\mathcal{K}_{\Gamma}\right) \subseteq \mathbb{H}^{3}$, each $\omega_{n}$ being based at a point $\left(z_{n}, t_{n}\right) \in \mathbb{H}^{3}$. Then the disk in the plane with center $z_{n}$ and radius $t_{n}$ intersects $\mathcal{K}_{\Gamma}$ - otherwise the hemisphere in $\mathbb{H}^{3}$ (hyperbolic halfplane) having the boundary of this disk as equator would separate $\left(z_{n}, t_{n}\right)$ from $\mathcal{K}_{\Gamma}$, so that $\left(z_{n}, t_{n}\right)$ would not belong to $\operatorname{ch}\left(\mathcal{K}_{\Gamma}\right)$. By Theorem 6.3 , there exists a holomorphic pair $\Gamma_{n}$ in $\mathcal{F}(\Gamma)$, whose fundamental annulus has conformal modulus uniformly bounded from below, such that $d\left(z_{n}, \mathcal{K}_{\Gamma_{n}}\right)=O\left(t_{n}\right)$ and $\operatorname{diam}\left(\mathcal{K}_{\Gamma_{n}}\right) \asymp t_{n}$. Conjugating $\Gamma_{n}$ by an affine Moebius transformation that takes $\omega_{n}$ onto a baseframe $\omega_{n}^{\prime}$ based at $(0,1) \in \mathbb{H}^{3}$, we obtain a new holomorphic pair $\Gamma_{n}^{\prime}$ with $d\left(0, \mathcal{K}_{\Gamma_{n}^{\prime}}\right)=O(1)$ and $\operatorname{diam}\left(\mathcal{K}_{\Gamma_{n}^{\prime}}\right) \asymp 1$. Since each $\omega_{n}^{\prime}$ differs from the standard baseframe at $(0,1)$ by an elliptic rotation about $(0,1)$ in $\mathbb{H}^{3}$, we deduce that $\Gamma_{n}^{\prime}$ belongs to $\left(\mathcal{F}(\Gamma), \omega_{n}\right)$ up to conjugation by such an elliptic rotation. It follows that any geometric limit of the form $\mathcal{G}=\lim \left(\mathcal{F}(\Gamma)^{\text {sat }}, \omega_{n}\right)$ contains the graph of a limiting holomorphic pair (possibly conjugated by a rotation of the Riemann sphere), and so each such $\mathcal{G}$ is twisting. Therefore $\left(\mathcal{F}(\Gamma), \mathcal{K}_{\Gamma}\right)$ is uniformly twisting as asserted.

Theorem 7.3. Let $\Gamma$ be a holomorphic pair with rotation number of bounded type, and let $H: \Gamma \simeq \Gamma^{\prime}$ be a quasiconformal conjugacy between $\Gamma$ and another holomorphic pair $\Gamma^{\prime}$. Then $H$ is $C^{1+\varepsilon}$-conformal at 0 , where $\varepsilon>0$ is universal.

Proof. By Lemma $7.2,\left(\mathcal{F}(\Gamma), \mathcal{K}_{\Gamma}\right)$ is uniformly twisting. Since by Theorem 5.1 the critical point of $\Gamma$ is $\delta$-deep for some $\delta>0$, the desired result follows at once from McMullen's inflexibility Theorem 7.1 applied to $\phi=H$ and $p=0$.

Corollary 7.4. Let $f$ and $g$ be real-analytic critical circle maps with the same rotation number of bounded type, and let $h: f \simeq g$ be the conjugacy that maps 
the critical point of $f$ to the critical point of $g$. Then $h$ has a quasiconformal extension which is $C^{1+\varepsilon}$-conformal at the critical point. In particular, the successive renormalizations of $f$ and $g$ converge together exponentially fast in the $C^{0}$ sense.

Proof. We know from Theorem 2.1 that $h$ is quasisymmetric. By Theorem 3.1, if $n$ is sufficiently large, then the $n$-th renormalizations $\mathcal{R}^{n} f$ and $\mathcal{R}^{n} g$ extend to holomorphic pairs $\Gamma_{n}(f)$ and $\Gamma_{n}(g)$, respectively. By the pull-back theorem for holomorphic pairs (Theorem 2.3), the restriction of $h$ to the long dynamical interval of $\Gamma_{n}(f)$ extends to a quasiconformal conjugacy between $\Gamma_{n}(f)$ and $\Gamma_{n}(g)$. But then Theorem 7.3 tells us that $H$ is $C^{1+\varepsilon}$-conformal at the critical point. The fact that $\mathcal{R}^{n} f$ and $\mathcal{R}^{n} g$ converge together at an exponential rate in the $C^{0}$ topology follows easily from this and Theorem 2.1.

The proof of Theorem 1.1 now follows from this corollary and the fact, proved in [3], that exponential convergence of renormalizations in the $C^{0}$-topology implies $C^{1+\alpha}$ conjugacy for some $0<\alpha<1$ depending on the rate of convergence. See also Th. 9.4 in [12], where a similar result is proved in the context of unimodal maps.

\section{ACKNOWLEDGMENTS}

We wish to thank D. Sullivan for sharing with us some of his deep insights on renormalization. We are grateful to $\mathrm{C}$. McMullen for explaining to us various aspects of his beautiful results on renormalization and rigidity.

\section{REFERENCES}

[1] L. Ahlfors, Conformal invariants, McGraw-Hill, 1973. MR 50:10211

[2] E. de Faria, Asymptotic rigidity of scaling ratios for critical circle mappings, Ergod. Th. \& Dynam. Sys. 19 (1999), 995-1035.

[3] E. de Faria and W. de Melo, Rigidity of critical circle mappings I, J. Eur. Math. Soc. 1.

[4] J. Graczyk and G. Świątek, Critical circle maps near bifurcation, Commun. Math. Phys. 176 (1996), 227-260. MR 96j:58053

[5] M. Herman, Sur la conjugaison differentiable des difféomorphismes du cercle a des rotations, Publ. Math. IHES 49 (1979), 5-234. MR 81h:58039

[6] Conjugaison quasi-simétrique des homéomorphismes du cercle a des rotations, Manuscript, 1988.

[7] L. Keen, Dynamics of holomorphic self-maps of $\mathbb{C}^{*}$, Holomorphic Functions and Moduli I, Eds. D. Drasin et al., Springer-Verlag, New York, 1988. MR 90e:58075]

[8] M. Lyubich and M. Yampolsky, Dynamics of quadratic polynomials: complex bounds for real maps, Ann. Inst. Fourier (Grenoble) 47 (1997), 1219-1255. MR 98m:58113

[9] C. McMullen, Complex dynamics and renormalization, Annals of Math. Studies 135 (1994). MR 96b:58097

[10] Renormalization and 3-manifolds which fiber over the circle, Annals of Math. Studies 142 (1996). MR 97f:57022

[11] Self-similarity of Siegel disks and Hausdorff dimension of Julia sets, Acta Math. 180 (1998), 247-292. MR 99f:58172

[12] W. de Melo and S. van Strien, One-dimensional Dynamics, Springer-Verlag, Berlin and New York, 1993. MR 95a:58035

[13] D. Sullivan, Bounds, quadratic differentials and renormalization conjectures, Mathematics into the Twenty-First Century, Amer. Math. Soc. Centennial Publication, vol.2, Amer. Math. Soc., Providence, RI, 1991. MR 93k:58194

[14] G. Świątek, Rational rotation numbers for maps of the circle, Commun. Math. Phys. 119 (1988), 109-128. MR 90h:58077 
[15] M. Yampolsky, Complex bounds for critical circle maps, Ergod. Th. \& Dynam. Sys. 19 (1999), 227-257. CMP 99:09

[16] J.-C. Yoccoz, Conjugaison analytique des difféomorphismes du cercle, Manuscript, 1989.

Instituto de Matemática e Estatística, Universidade de São Paulo, Rua do Matão 1010, CEP05508-900 SÃo PaUlo SP - BRasil

E-mail address: edson@ime.usp.br

Instituto de Matemática Pura e Aplicada, Estrada Dona Castorina 110, Jardim BotÂNico, CEP22460-320 Rio de JANEIRo RJ - BRASIL

E-mail address: demelo@impa.br 